\title{
Analysis on Mitigation of Intelli-drive Communication Congestion: Broadcasted Signal Phase in Vehicle-to-Vehicle (V2V) Safety Features Using DSRC IEEE 802.11p Standard
}

\author{
Richmond Adebiaye* \\ Information Systems, College of Business and Technology, Parker University, Dallas Tx, United States \\ *Corresponding author: richmond.adebiaye@yahoo.com
}

\begin{abstract}
This study explores functionalities of the Dedicated Short Range Communication architecture, and provides V2V safety information systems which provide warnings on crash avoidance. An application (DSRC) of traffic safety is tested for congesting. This study is based on Short Range Communication (DSRC) standard, which has become an emerging vehicular safety application. A MAT-LAB simulation experiment is performed using wireless spectrum, layering of routing protocols to determine the ability to mitigate vehicle to vehicle communication congestion within Vehicle-to-Vehicle (V2V) signal phase communications. The objective was to have a support transition from a prototype-level to a deployment-level system.
\end{abstract}

Keywords: DSRC architecture, vehicle-to-vehicle signals, vehicle safety, crash avoidance, IEEE 802.11p

Cite This Article: Richmond Adebiaye, "Analysis on Mitigation of Intelli-drive Communication Congestion: Broadcasted Signal Phase in Vehicle-to-Vehicle (V2V) Safety Features Using DSRC IEEE 802.11p Standard." American Journal of Computing Research Repository, vol. 5, no. 1 (2017): 1-13. doi: 10.12691/ajcrr-5-1-1.

\section{Overview of DSRC Study}

Transportation applications could be implemented for safety architecture or communication protocols. It also provides analysis of vehicle-to-vehicle (V2V) safety systems using the adopted Dedicated Short Range Communication (DSRC) standard, which has become an emerging vehicular safety application. The functions of DSRC architecture help to assess vehicle to vehicle safety information systems that provide safety warnings on crash avoidance or accidents. Dedicated Short Range Communication (DSRC) is wireless technology which can operate at $75 \mathrm{MHz}$ of spectrum at frequency of $5.9 \mathrm{GHz}$ as allotted by the 'Federal Communications Commission' (FCC). The reduction of congestion is most important in order to avoid collision.

DSRC would "support safety-critical communications especially collision warnings, as well as other valuable ITS applications such as Electronic Toll Collection (ETC), digital map update, etc.” [13]. One researcher also noted that the "versatility of DSRC greatly enhances the likelihood of its deployment by various industries and adaptation by the consumers". [13]

The traffic flow improves and this leads to the reduction of congestion and ultimately collision of vehicles is reduced. Safety technology has always been an important concept of vehicle manufacturers and this has developed rapidly since NHTSA began regulating the auto industry. According to studies by NHTSA, it is believed that "vehicles protect occupants much better in the event of a crash due to advanced structural techniques propagated by more stringent crashworthiness standards, and some crash avoidance technologies are now standard equipment" [1].

Looking at vehicular crashworthiness and required crash avoidance technologies, researchers like $[5,6]$ believed that motor vehicles are safer now than before. However, a "significant number of annual crashes remains that could potentially be addressed through expanded use of more advanced crash avoidance technologies" [2]. Even at this rate of annual crashes, it is generally believed that technology can help drivers avoid crashes. The purpose of this study is to assess safety communication of vehicle-tovehicle features and test this communication technologies using a simulation experiments configured on wireless network at the routing layer to test the ability to mitigate $\mathrm{V} 2 \mathrm{~V}$ communication congestion which has always been an impediment to the implementation that support transition from a prototype-level to a deployment-level system.

\subsection{Importance of Connected Vehicle Using Simulation Techniques}

The simulation software provides the data for designing reliable and good safety. By simulation, prototype can be developed. In order to determine the optimal system parameters and components of DSRC systems before the actual implementation, "reliable performance evaluation methods are needed by system designers as well as system operators" [10]. In another research by [11], explained 
thoroughly "the interference between different services, the optimal control parameters for the communication protocols, the usage ratio of the communication zone and the influence of physical link characteristics (such as windscreen attenuation)" are important to safety standards. This technology "was established for services involving vehicle-to-infrastructure (V2I) and vehicle-to-vehicle (V2V) communications”[12].

For the purpose of this study, the vehicle-to-vehicle (V2V) is analyzed. It was indicated in the "Standards Committee E17.51 which endorses a variation of the IEEE 802.11a MAC for the DSRC link", that also that "DSRC supports vehicle speeds up to $120 \mathrm{mph}$, nominal transmission range of $300 \mathrm{~m}$ (up to $1000 \mathrm{~m}$ ), and default data rate of $6 \mathrm{Mbps}$ (up to 27Mbps)"[3].

The objectives of even adopting this technology will provide two-way advantage - to vehicle manufacturer and to drivers. According to [6], "the technology could warn drivers of impending danger so that the driver can take corrective action, or may even be able to intervene on the driver's behalf”, thereby, reducing deaths, injuries, and economic losses resulting from motor vehicle crashes by setting and enforcing safety performance standards for motor vehicles and motor vehicle equipment [5]. It also helps vehicle manufacturers in building safer vehicles.

Variables to test will include vehicular safety performances and the learning orientation for high-tech safety measures while the independent variables include new safety measures and improved technology.

\section{Problem Statement}

In present era, traffic has increased to high level. Accidents occur due to collisions and crashes between vehicles which result in the loss of lives. So an invention is needed which can avoid these types of accidents and loss of lives. Keeping this in mind, working on Dedicated Short Range is in progress [3]. Modern transportation system especially in the interstate system has brought about increased traffic congestion. Road accidents and injuries occur regularly due to crashes and expensive impacts on air quality. As a result of this, it is pertinent for new paradigm invention to solve safety issues, environment and congestion. It is also necessary to apply advanced technology to current infrastructure using various computing mechanisms and sensors, in order to increase the operational capacity of the systems and achieve better overall transportation network operations [6].

\section{Research Methodology}

In this study, an application of Dedicated Short Range Communication (DSRC) standard on traffic safety was experimented using MAT-LAB analysis of vehicle-tovehicle (V2V) safety. This study uses simulation-based research to experiment application of $\mathrm{V} 2 \mathrm{~V}$ communications technology, in order to test the mitigating factors on V2V communication collisions based on the complexity of the DSRC applications. In this context, a simulation can be defined as 'a method for using computer software to model the operation of "real-world" processes, systems, or events' [9]. Other researchers [3] also analyzed how in simulation studies of this kind, "the researcher develops a model of the phenomenon under investigation and then chooses an appropriate simulation method" (pg. 481).

MAT-LAB was used for simulation. In the coding, one of the vehicles will be related to as either a 'master' or as a 'slave', in order to determine the speed change as vehicles cross on the roads. This represents a simulation based research in which $\mathrm{V} 2 \mathrm{~V}$ communication technology is tested, in order to mitigate $\mathrm{V} 2 \mathrm{~V}$ communication congestions which represents one of the problems inherent in DSRC. The ability to mitigate this congestion allows for safety features of reducing collisions in vehicles on the road. Keeping this in mind, "simulation is a method in which analysis of real life is to be done on software. Therefore, by choosing appropriate method, simulation models are obtained" [9].

Two roads crossing each other at different angles are shown in different scenarios, representing vehicles crossing each other using MAT-LAB simulation. The simulation concept integrates:

a. Both microscopic traffic and realistic models of the communication channel which also include implementation of the DSRC architecture,

The simulation also shows that "the new DSRC offers the potential to effectively support vehicle-to vehicle and vehicle-to-roadside safety communications, which has become known as Vehicle Safety Communication (VSC) technologies"[6].

The limitation of this study is the inability to use Matlab to create 'DSRC Carsim” in the wireless network layer to pin-point the DSRC point of communication

\section{Routing Protocols}

The state of the art in Inter-vehicular communication (IVC) is experimented using frequency range within DSRC $5.9 \mathrm{GHz}$ as allocated. Communication via satellite general positioning systems (GPS) is proposed as antenna links for all vehicle communications; since vehicles are moving objects, so volatile link quality is needed. Moreover reactive on demand protocols are used as these are activated when needed. Time-Division Multiple Access (TDMA), Frequency-Division Multiple Access (FDMA) and Code-Division multiple Access (CDMA) are all difficult to use in this experiment, as these are best for still application [1]. So the "multiple access protocol at the datalink layer which proposes how multiple terminals access the medium without interference or collision" known as 'ALOHAnet' and Carrier Sense Multiple Access (CSMA) are best protocols used and as our communication is broadcast and not unicast, the use of Request to send and Clear to send (RTS/CTS) is beyond the implementation plan in this experiment.

In real life, the High Performance Radio LAN which is the Wireless LAN Standard and the European alternative for the IEEE 802.11 standards (HIPERLAN) and the black burst which is the analog signal used in broadcasting are designed to support quality of service across IP networks which is an increasingly important aspect of Enterprise IT infrastructure (QoS). But these two protocols have no method to combat the hidden terminals 
Using the Contention-based Channel Access, which is the Enhanced Distributed Coordination Function (EDCF), performance can be evaluated by increasing the overall system throughput, allow the probability of collision to be large. This represents the choice of Distributed coordination function (DCF), which is the fundamental MAC technique of the IEEE 802.11 based WLAN standard, thus allowing for attaining quality of service (QoS) [8]. This does not indicate that quality of service (QoS) will also be attained with accuracy.

In view of this, a proper alteration is needed for QoS in real life scenario of transmission and broadcast. For example, each car has its own IP address on which it is identified. Then vehicles send their information via the IP address using the DSRC standard to establish communications. Such scenario was implemented in this study. DSRC 'SIM card' was not developed or experimented but assumptions were made to determine its functionalities.

\section{Experiment (MAT-LAB Simulation) Setup}

The experiment shows the coding and formulas that simulated the DSRC technique. Screenshots imagery are also analyzed.

\subsection{Coding Process}

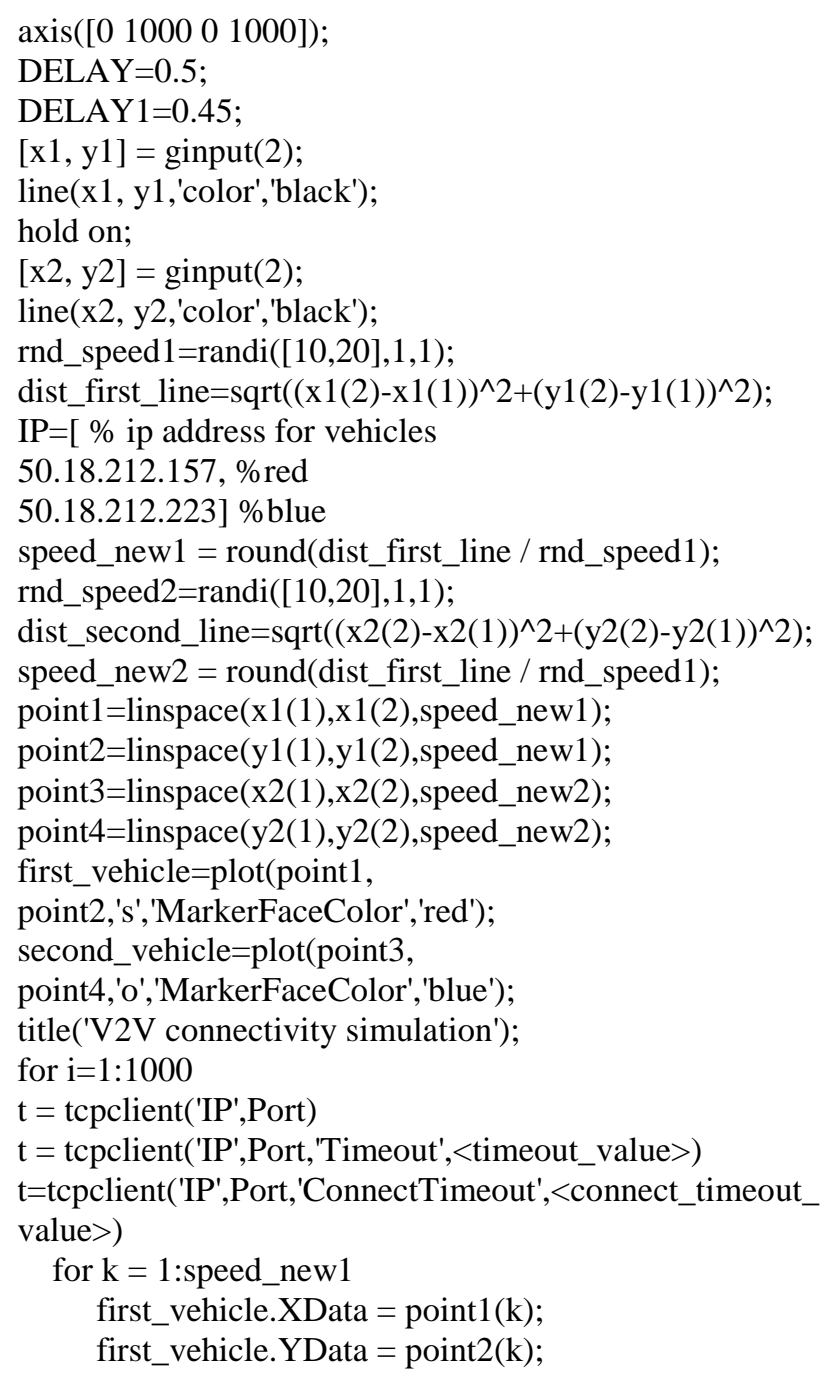

second_vehicle.XData $=$ point $3(\mathrm{k})$;

second_vehicle. YData $=$ point $4(\mathrm{k})$;

plot(point1(k),point2(k),point3(k),point4(k));

vehicle_dist=[point1(k), point2(k);point3(k),

point4(k)];

distance1= pdist(vehicle_dist,'euclidean');

If distance $1<=100$

line $1=\operatorname{plot}([\operatorname{point} 1(\mathrm{k})$,

point3(k)],[point2(k),point4(k)],'--','color','green');

pause(0.3);

set(line1,'Visible','off');

batchFiles = dir('PathToBatchfiles ${ }^{*} .{ }^{*}$ ');

for $\mathrm{i}=1$ :length(Routing)

run(fullfile('Routing', batchFiles(i).Routing));

end

Routing [NewEvents] = action(50.18.212.157, 12);

Routing [NewEvents] = action $(50.18 .212 .223,13)$;

end

pause(DELAY1);

$\mathrm{t}=$ tcpclient('50.18.212.157', 12,'ConnectTimeout', 30)

t = tcpclient('50.18.212.223 ', 13,'ConnectTimeout', 30)

end

set(first_vehicle,'Visible','off');

set(second_vehicle,'Visible','off');

end

hold off;

\subsection{Formulas, Data and Key Variables of Code}

1. To set the window size to 1000 the formula is made as follows

axis([0 100001000$])$;

2. To take data or input from user for line 1 , this is provided by user at start of simulation

$$
[\mathrm{x} 1, \mathrm{y} 1]=\operatorname{ginput}(2) \text {; }
$$

3. To draw the line (Road 1) Data provided for road 1 line(x1,y1,'color','black');

4. To take input from user for line 2 line, this is also provided by user at start

$$
\text { [x2,y2] = ginput(2); }
$$

5. To draw the line (Road 2) Data provided for road 2

6. For all the values in linspace (x2,y2,'color','black');

$$
\text { for } \mathrm{k}=1 \text { :speed_new1 }
$$

7. First vehicle's $x$ co-ordinate/X-coordinate data first_vehicle.XData = point1 $(\mathrm{k})$;

8. First vehicle's y co-ordinate/Y-coordinate data first_vehicle.YData = point $2(\mathrm{k})$;

9. Second vehicle's $\mathrm{x}$ co-ordinate/X-coordinate data second_vehicle.XData $=$ point $3(\mathrm{k})$;

10. If the distance is within $200 \mathrm{~m} /$ distance variable to check whether vehicles are in the radius of 100 meter or not

$$
\text { if distance } 1<=100
$$

11. Show connectivity between two vehicles and pause of $0.45 /$ Data or formula used to show connectivity on simulation

line1=plot([point1(k),point3(k)],[point2(k),point4(k)]

,'--','color','green'); pause(0.3); set(line1,'Visible','off'); end pause(DELAY1); 
12. Load the routing file which rubs for loop

13. These are used to route the MAT-LAB functions with IP Routing layer is formed due to which it tests the ability to mitigate V2V communication congestion. $\mathrm{t}=$ tcpclient('50.18.212.157', 12, 'ConnectTimeout', 30) t = tcpclient('50.18.212.223 ', 13, 'ConnectTimeout', 30) batchFiles $=\operatorname{dir}\left(\right.$ 'PathToBatchfiles $\left.{ }^{*} . \mathrm{m}^{\prime}\right)$; for $\mathrm{i}=1$ :length(Routing) run(fullfile('Routing', batchFiles(i).Routing)); end

14. Defines IP and port number of each car Routing [NewEvents]= action(50.18.212.157, 12); Routing [NewEvents] = action $(50.18 .212 .223,13)$;

\subsection{Table 1: Showing Vehicle Positions and Their IP Addresses}

In Table 1, data is analyzed including vehicles positions and their IP addresses are shown.

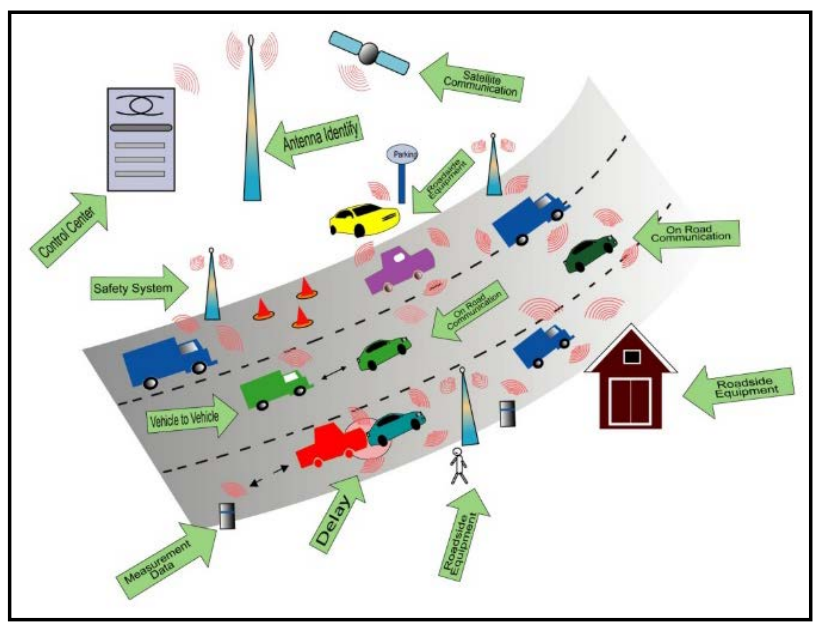

Figure 1. Simulation Results

\section{Vehicle Mobility Performance Comparison}

In the result analysis, we discussed four case scenarios to analyze our simulation concept.

\subsection{Vehicles Cross at Some Angle}

In first case, vehicles are crossing at some level. In simulation when vehicles get closer to each other, then one of them, depending upon their speed, becomes master and other becomes slave, speed of the slave vehicle becomes slower than that of master and the vehicles crossed each other without colliding or collusion.

In Figure 2 below, our simulation shows two vehicles with blue and red approaching each other. In Figure 3, below is the screenshot. From the image, it can be seen that speed of blue car decreases and that of the red car increases as it crosses the road without collision.

\subsection{Vehicles Cross at 35 Angle}

In this simulation case, the vehicles crossed at an angle of $35^{\circ}$. The scheme is assumed to be constant, as the red and blue vehicles approach each other. While one of them becomes the slave, the other will become the master. The dotted line shows the communication between two vehicles when they come closer to each other. The blue car enhances its speed as it becomes the master. (Figure 7Figure 11)

\subsection{Vehicles Cross at 90 Angle}

In this case the vehicles will cross each other at $90^{\circ}$. In figures below the V2V Dedicated Short range Communication allows these vehicles to connect wirelessly with each other. (Figure 12-Figure 15)

\subsection{Vehicles Cross at 180 Angle}

In the case below, the two vehicles are moving parallel to each other. Vehicles communicate but moving at constant speed. None becomes slave or master as there are no chances of collision. (Figure 16-Figure 24).

Table 1. Data used in simulation for connectivity of vehicles using DSRC and IP addresses in this simulation IP addresses are stored in the addresses as shown in table

\begin{tabular}{|c|c|c|c|c|}
\hline Latitude & Longitude & Steps number & Address & IP addresses \\
\hline 60.2157 & 55.4808 & 4 & \#31b & 50.18.212.157 \\
\hline 4.81667 & 8.23333 & 8 & \#01b & 50.18.212.223 \\
\hline-43.0375 & -19.75 & 97 & \#68e & 50.18.212.157 \\
\hline-98.7639 & 19.0921 & 47 & \#ес2 & 50.18.212.223 \\
\hline-49.3 & -28.71333 & 78 & \#5d6 & 50.18.212.157 \\
\hline 111.8892 & -8.1318 & 2 & \#908 & 50.18.212.223 \\
\hline 22.58394 & 52.26816 & 3 & \#21f & 50.18.212.157 \\
\hline 22.6914 & 51.54409 & 58 & \#511 & 50.18.212.223 \\
\hline 122.74754 & 40.85158 & 60 & \#601 & 50.18.212.157 \\
\hline 2.5627 & 48.8498 & 39 & \#56e & 50.18.212.223 \\
\hline 72.01928 & 33.42985 & 47 & \#f1d & 50.18.212.157 \\
\hline 113.11673 & 23.11974 & 19 & $\# 02 \mathrm{a}$ & 50.18.212.223 \\
\hline 18.3898 & 59.3268 & 60 & \#7a5 & 50.18 .212 .157 \\
\hline 29.84711 & 62.76023 & 33 & \#59d & 50.18.212.223 \\
\hline 113.66499 & 37.61469 & 81 & \#826 & 50.18.212.157 \\
\hline-103.1275 & 21.405 & 30 & \#cес & 50.18.212.223 \\
\hline 109.9025 & -7.36 & 49 & \#fb8 & 50.18.212.157 \\
\hline 117.30725 & 52.82657 & 55 & $\# 0 \mathrm{a} 2$ & 50.18.212.223 \\
\hline-8.5616 & 40.2261 & 19 & \#b29 & 50.18.212.157 \\
\hline 129.45305 & 49.09356 & 99 & \#772 & 50.18.212.223 \\
\hline-12.03333 & 9.75 & 61 & \#f1d & 50.18.212.157 \\
\hline-64.49 & -27.16607 & 79 & \#05b & 50.18.212.223 \\
\hline 19.79194 & 42.73389 & 48 & \#98b & 50.18.212.157 \\
\hline 24.05571 & 57.03132 & 88 & \#d86 & 50.18.212.223 \\
\hline 13.46948 & 50.09703 & 68 & \#d49 & 50.18.212.157 \\
\hline 32.69639 & 55.06667 & 94 & \#c23 & 50.18.212.223 \\
\hline 105.15464 & 15.3157 & 93 & \#c48 & 50.18.212.157 \\
\hline 11.06056 & 35.50056 & 49 & $\# 2 \mathrm{fb}$ & 50.18.212.223 \\
\hline 48.68722 & 63.41064 & 65 & \#71a & 50.18.212.157 \\
\hline 108 & 13.98333 & 37 & \#0b1 & 50.18 .212 .223 \\
\hline-61.08161 & -27.08966 & 80 & \#11b & 50.18.212.157 \\
\hline-51.945 & -29.40111 & 29 & \#ba7 & 50.18 .212 .223 \\
\hline-62.76667 & 17.3 & 36 & \#ccb & 50.18.212.157 \\
\hline 139.6 & 36.06667 & 70 & \#803 & 50.18.212.223 \\
\hline
\end{tabular}




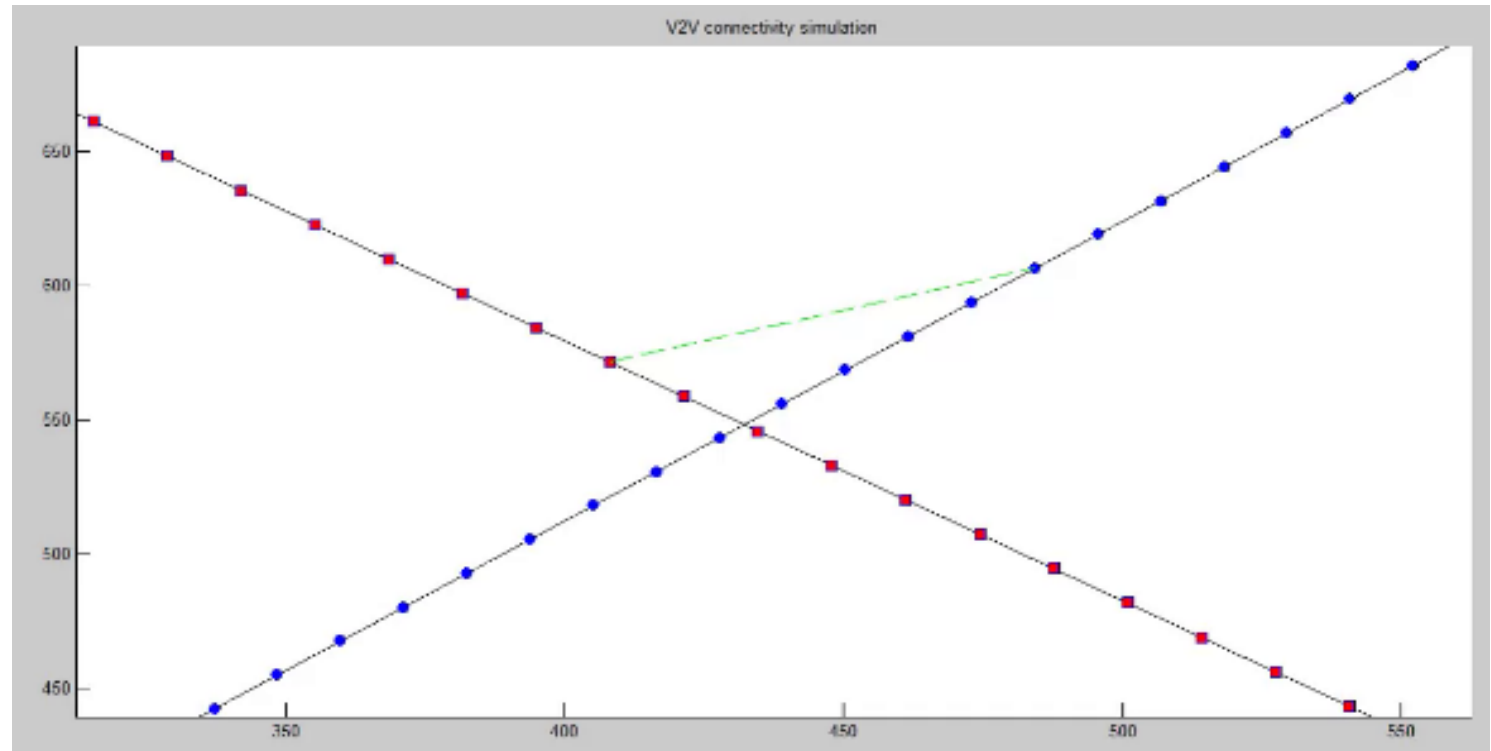

Figure 2. Two vehicles blue and red are moving as they establish communications using the simulated Dedicated Short Range communication System standard (DSRC). *No DSRC CarSim created*

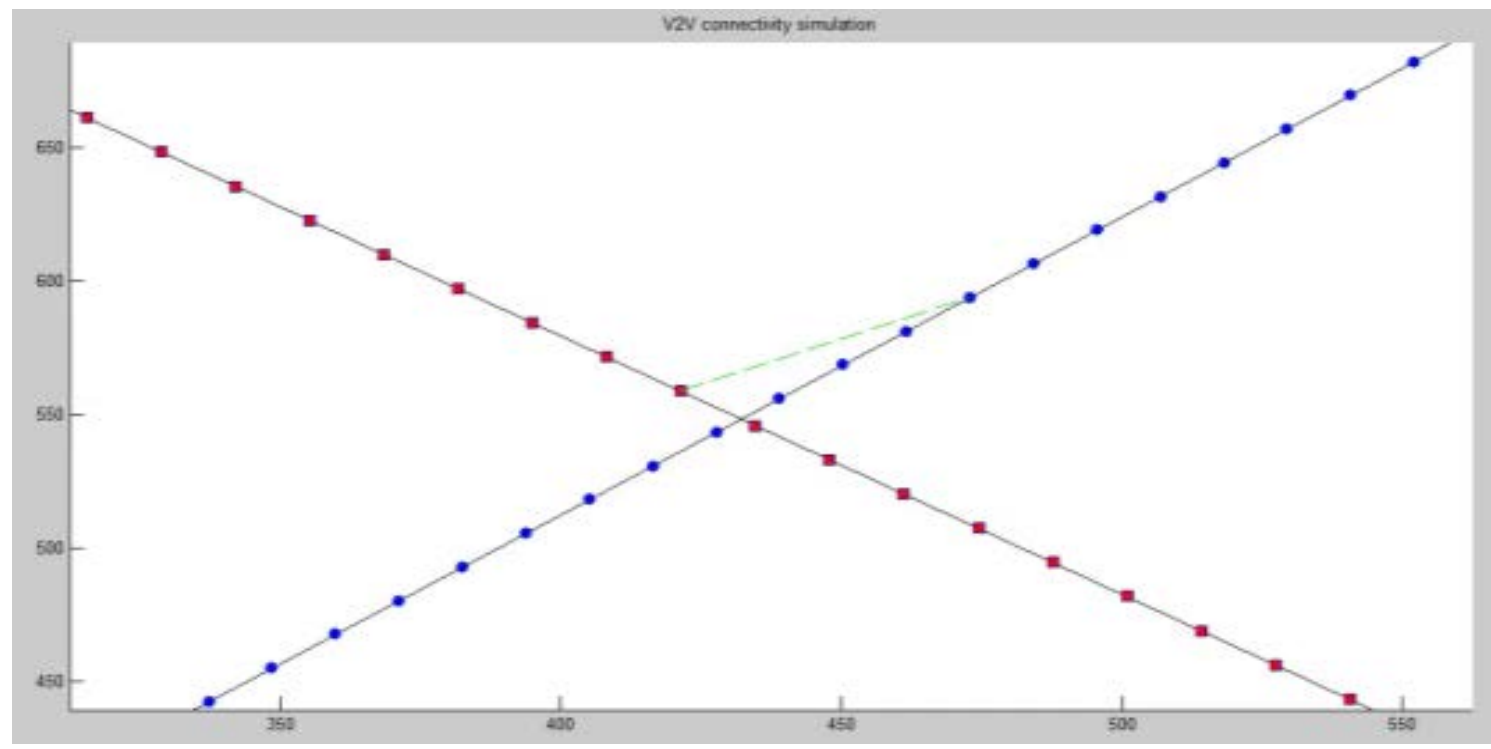

Figure 3. Simulation shows how the blue car reduces speed while Red vehicle increases speed

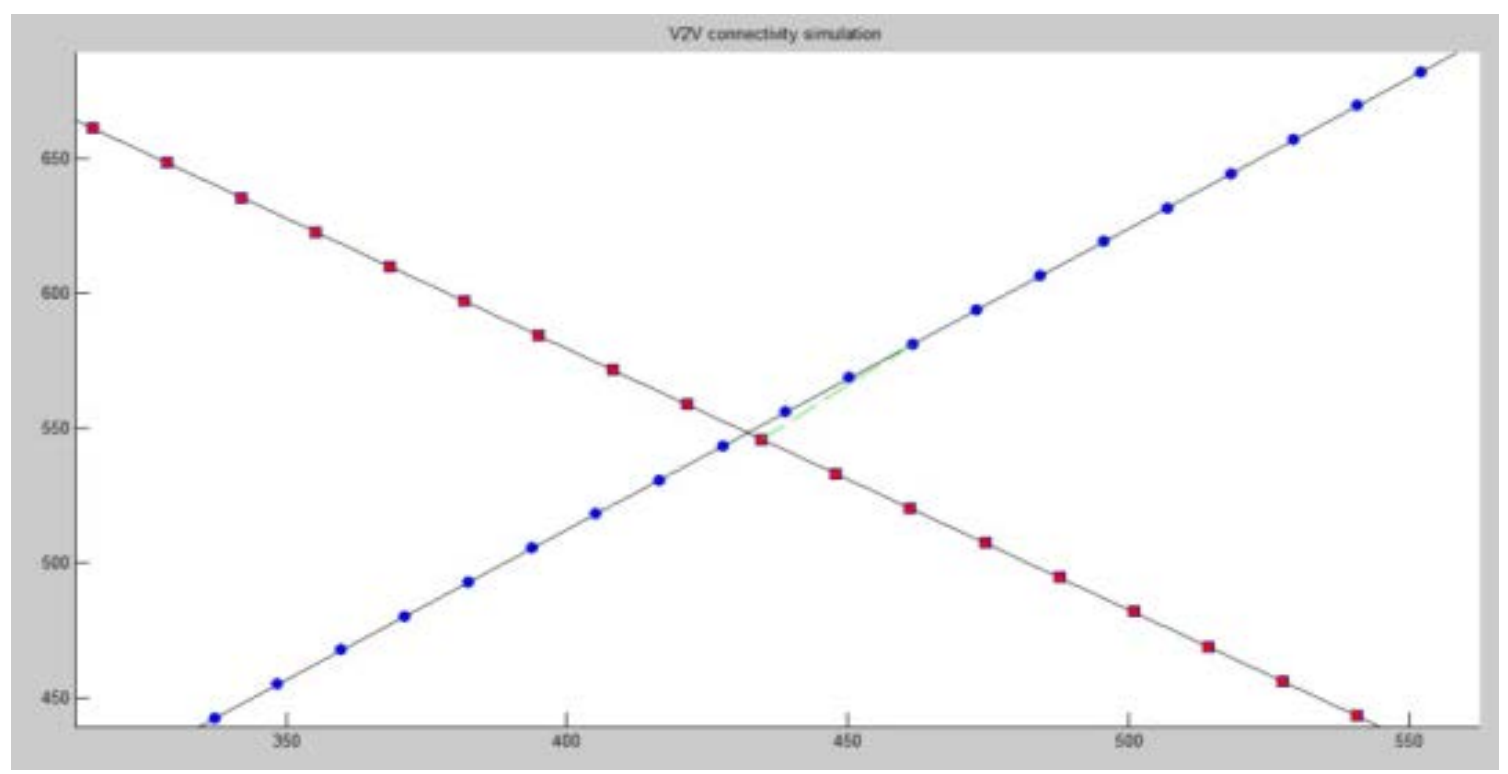

Figure 4. The screenshot shows how the red vehicle moves before blue vehicle without collision. 


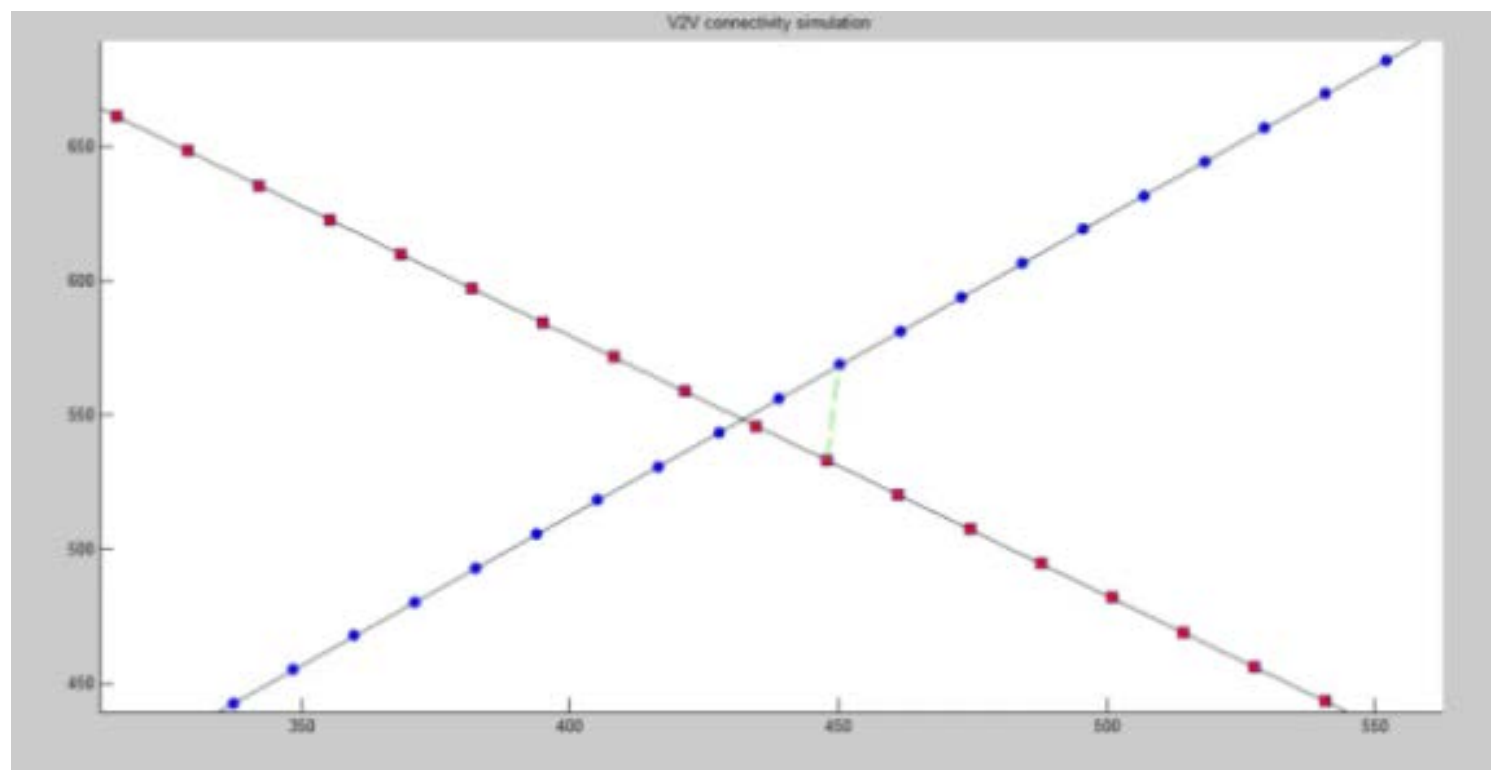

Figure 5. Both vehicles are still connected using the DSRC standard

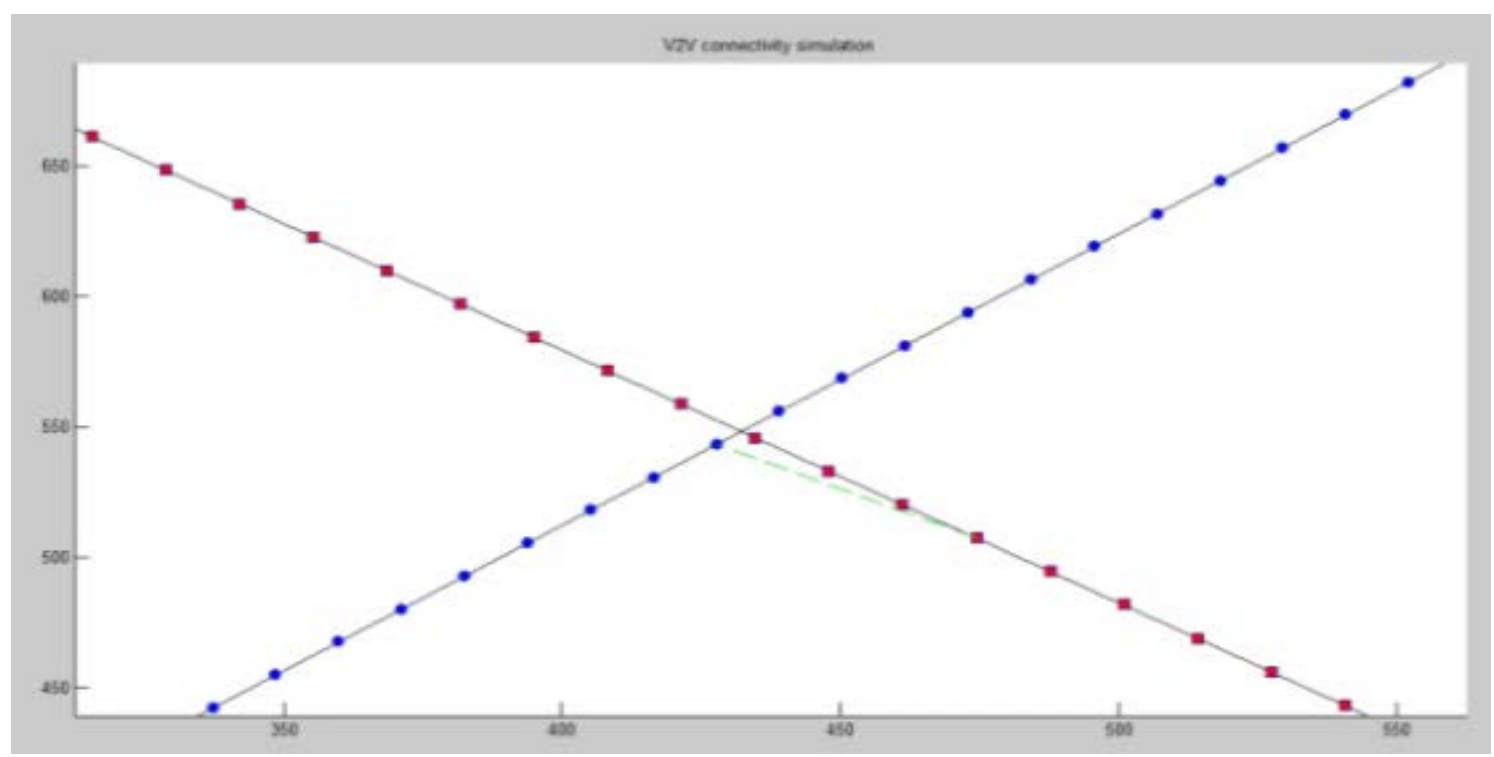

Figure 6. Now, both vehicles maintain their previous speeds but are still connected

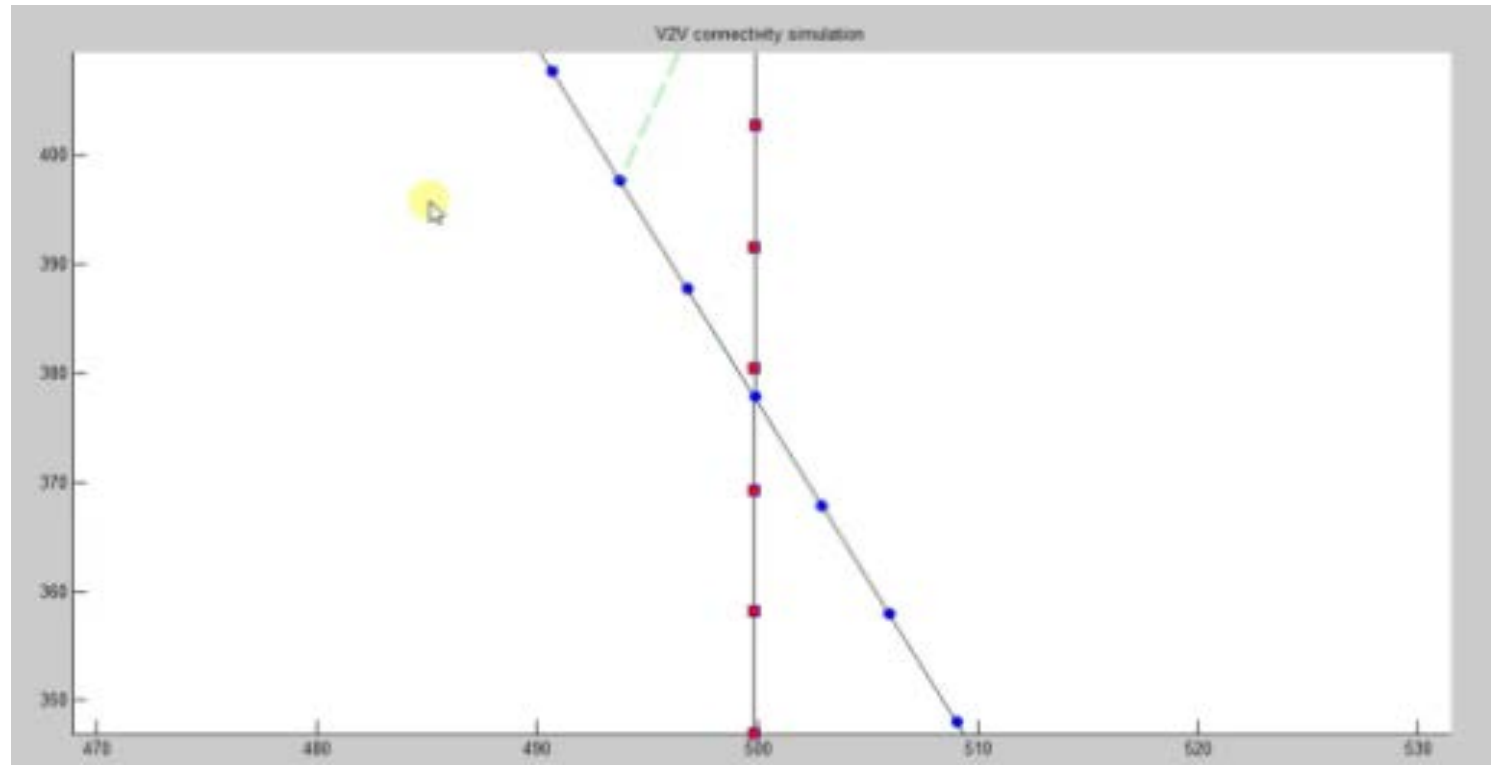

Figure 7. Red and blue vehicles are on the road and have to cross at an angle of $35^{\circ}$. 


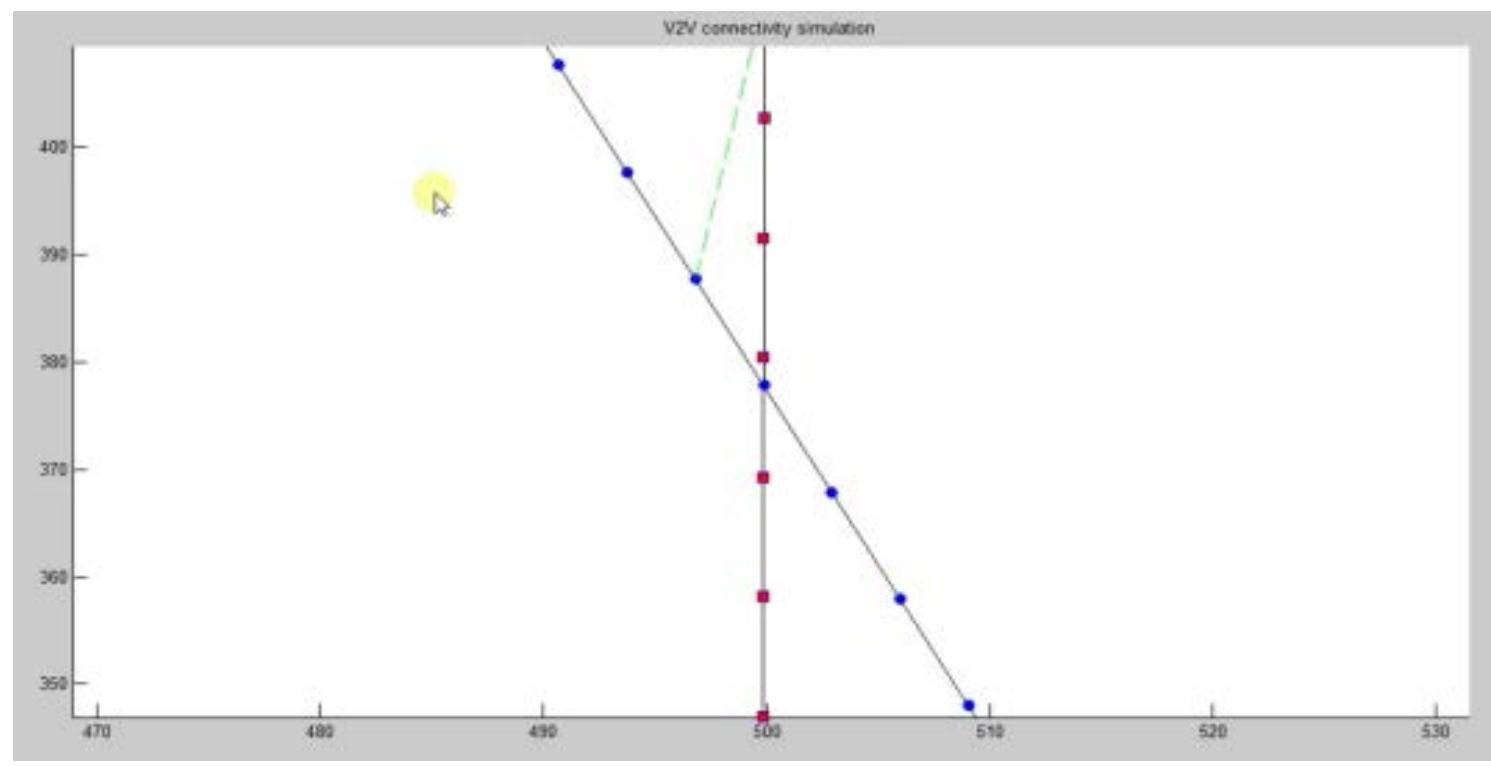

Figure 8. Blue car reduces its speed by $5 \mathrm{~ms}$ per meter, while the red car increases by $5 \mathrm{~ms}$ per meter

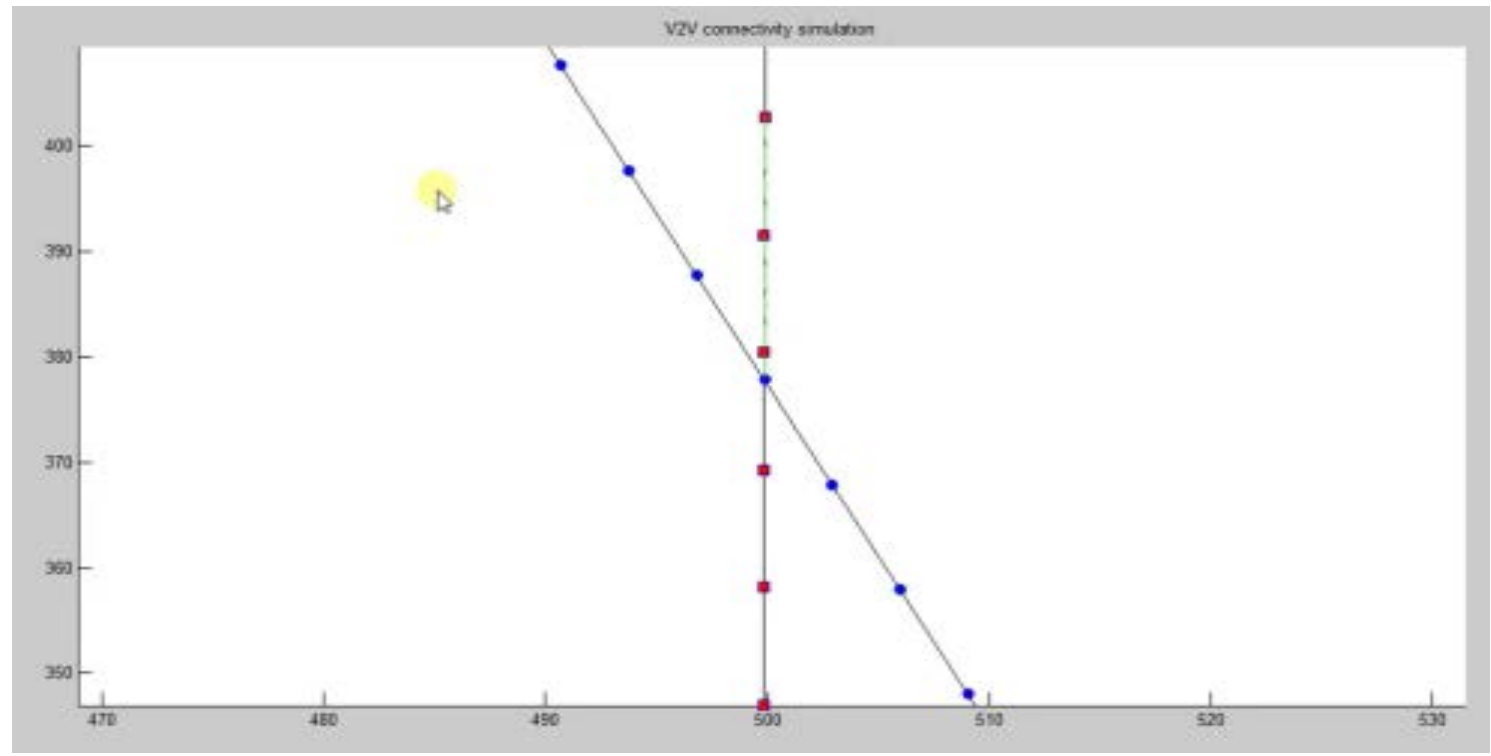

Figure 9. Blue car crossed the road as its speed was faster

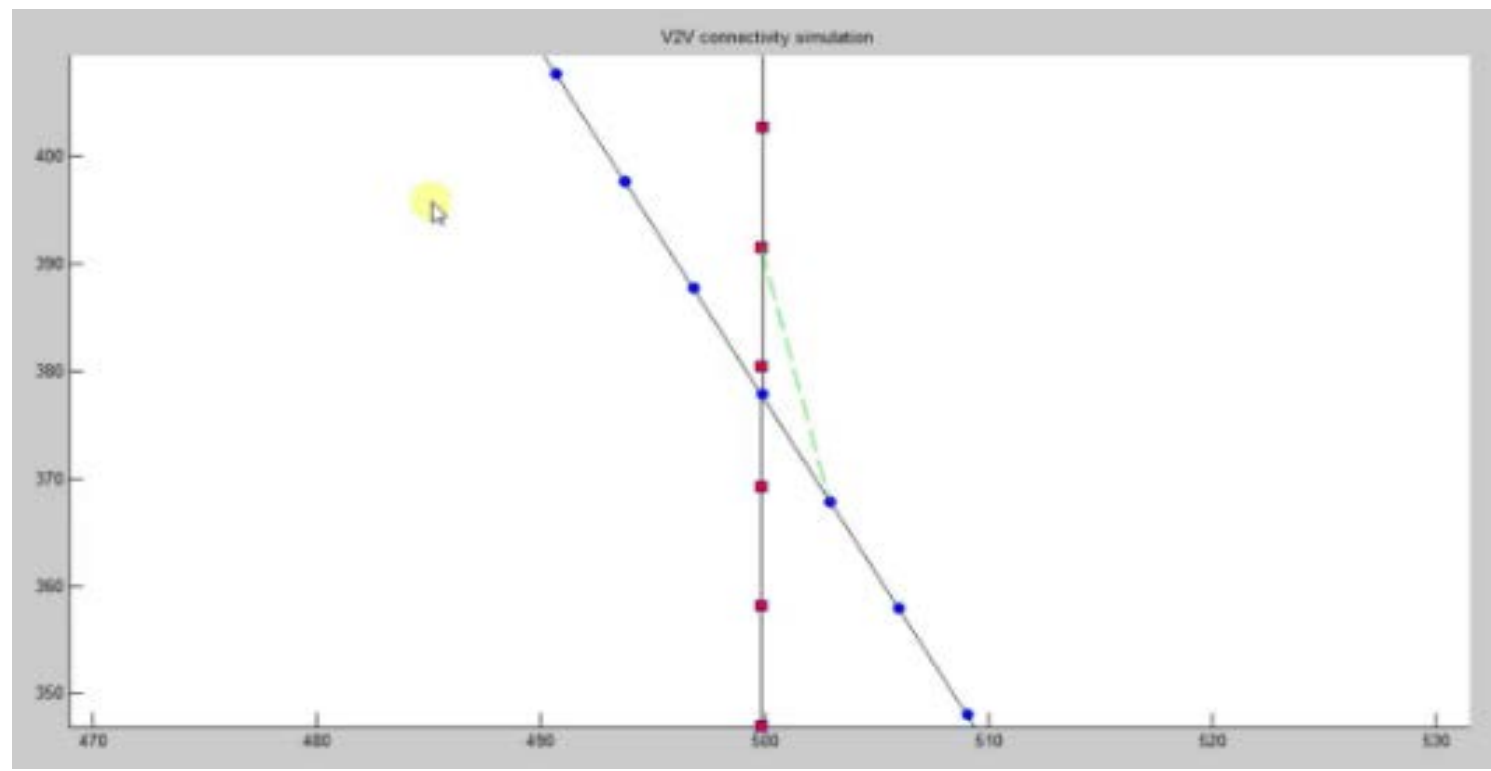

Figure 10. After crossing, the vehicles are still connected. 


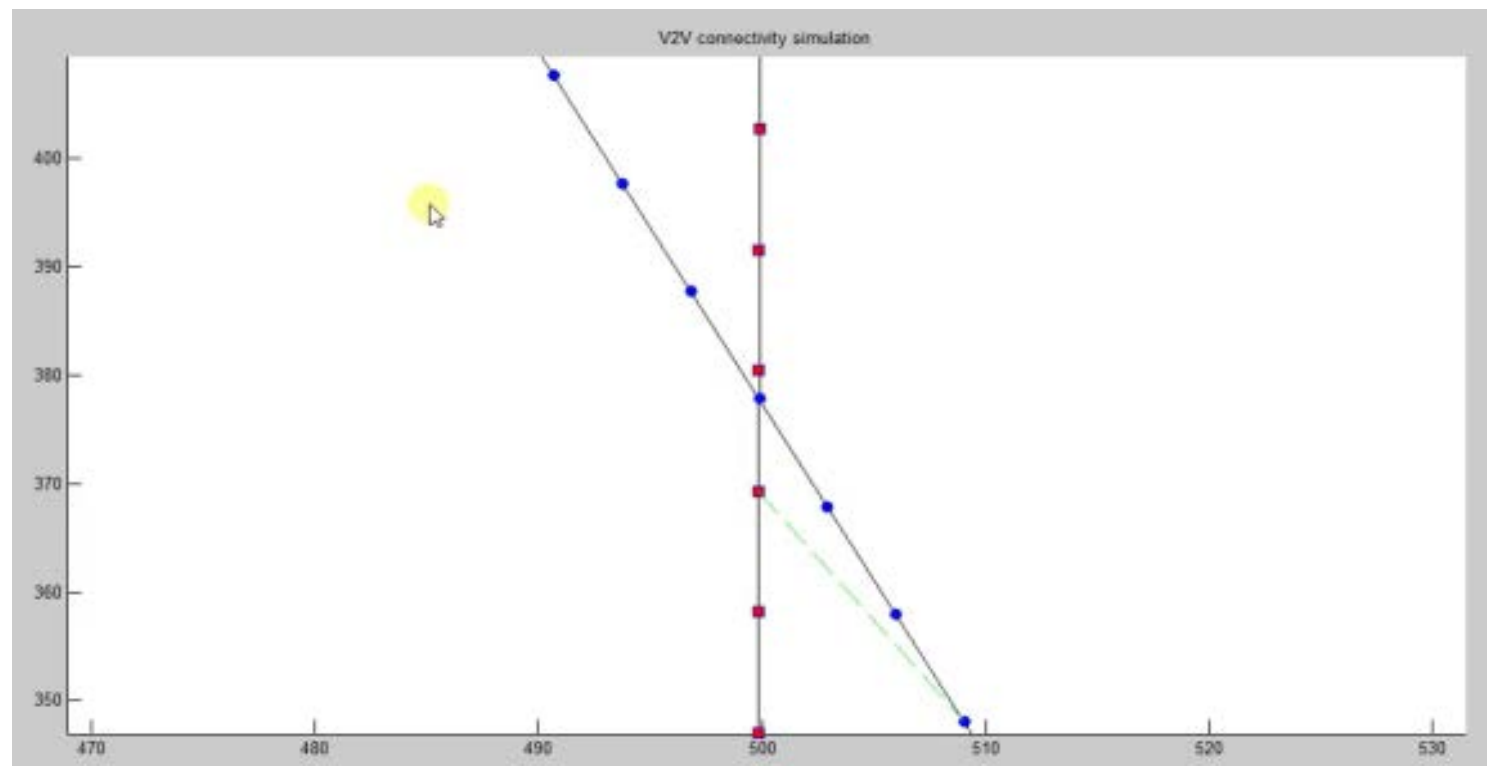

Figure 11. Simulated to show both vehicles passed avoiding collision

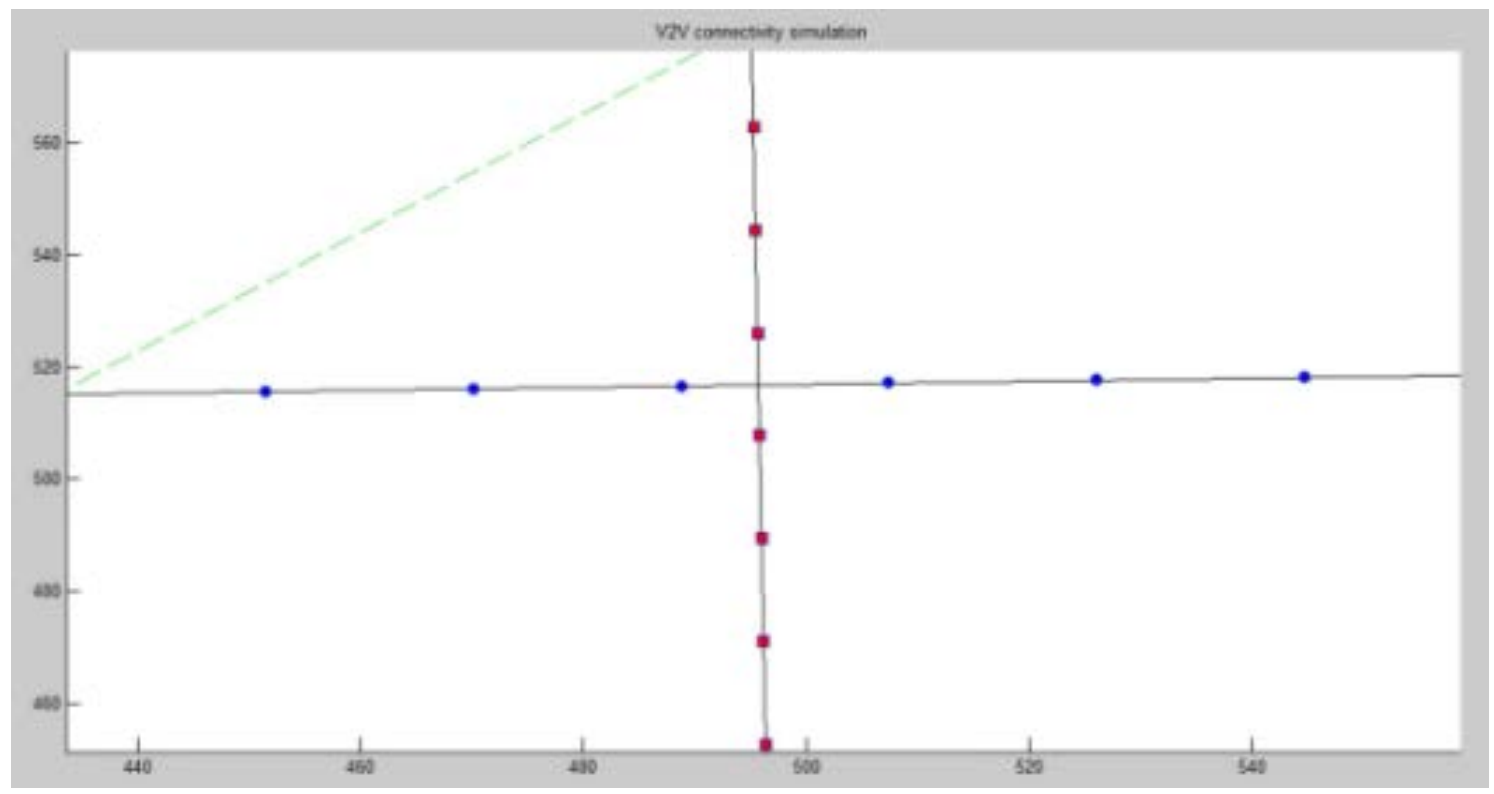

Figure 12. Now, blue and red vehicles have to pass each other on road at 90 degrees

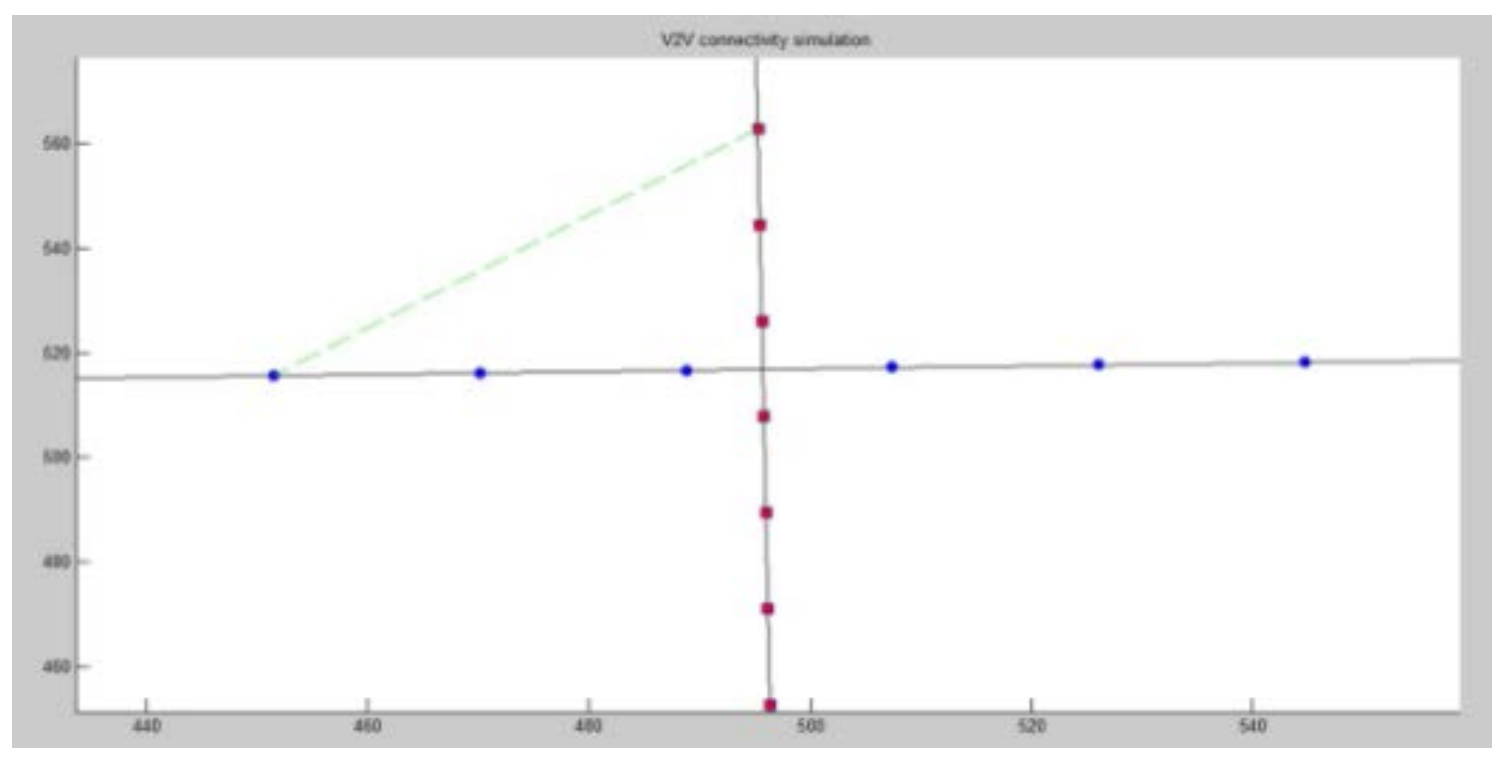

Figure 13. Simulation shows vehicles approaching each other 


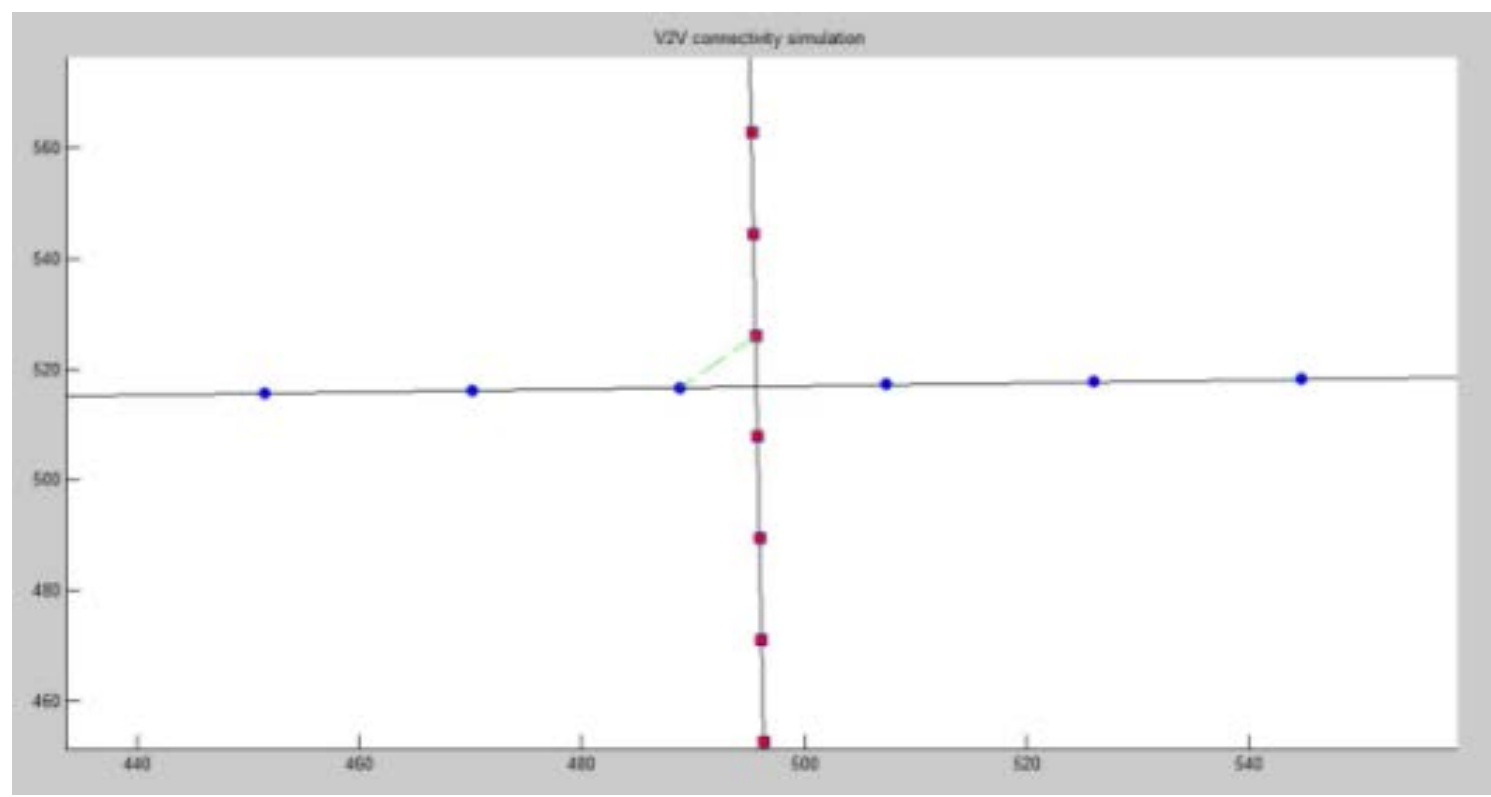

Figure 14. Red car reduces its speed and Blue enhances the speed

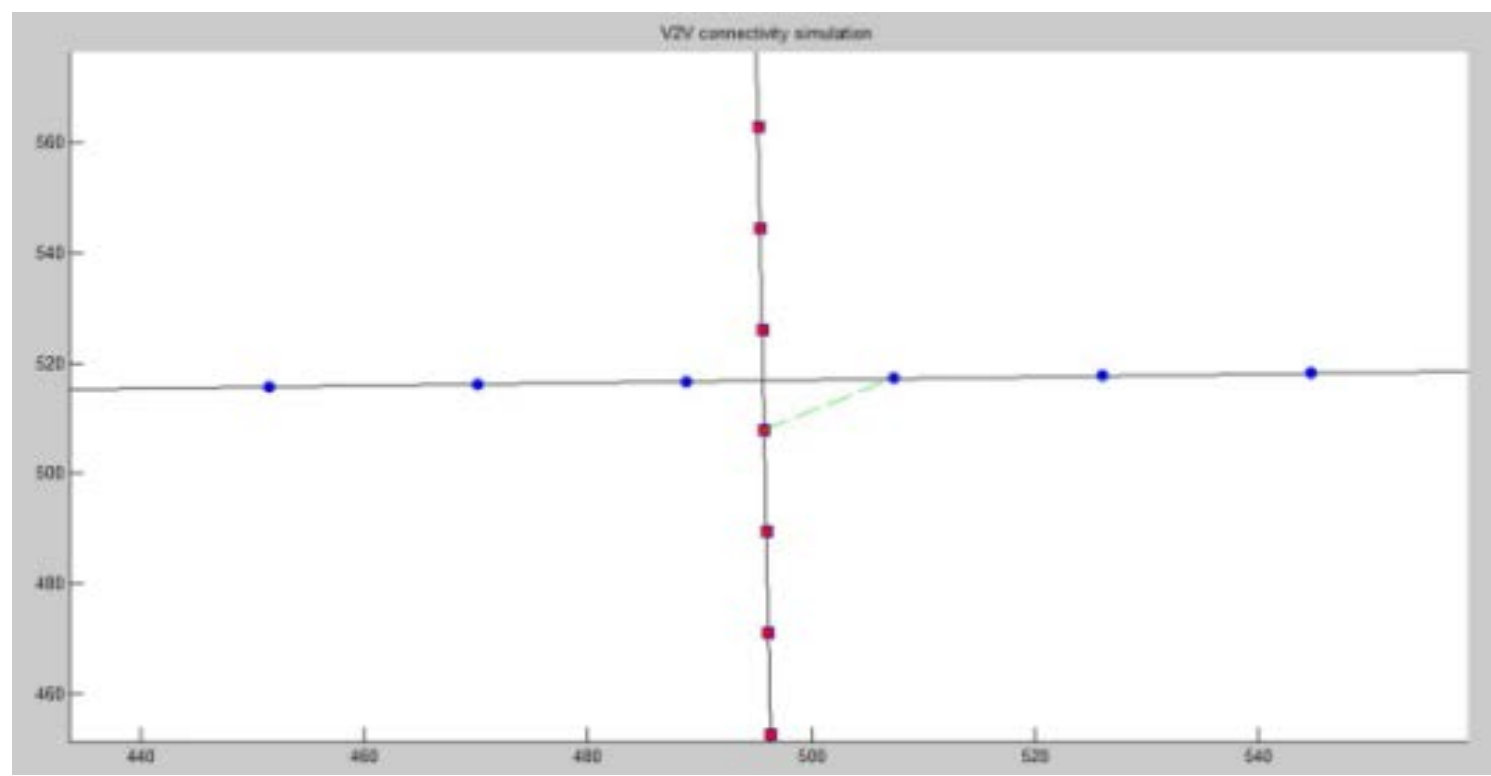

Figure 15. Both of the vehicles have now passed

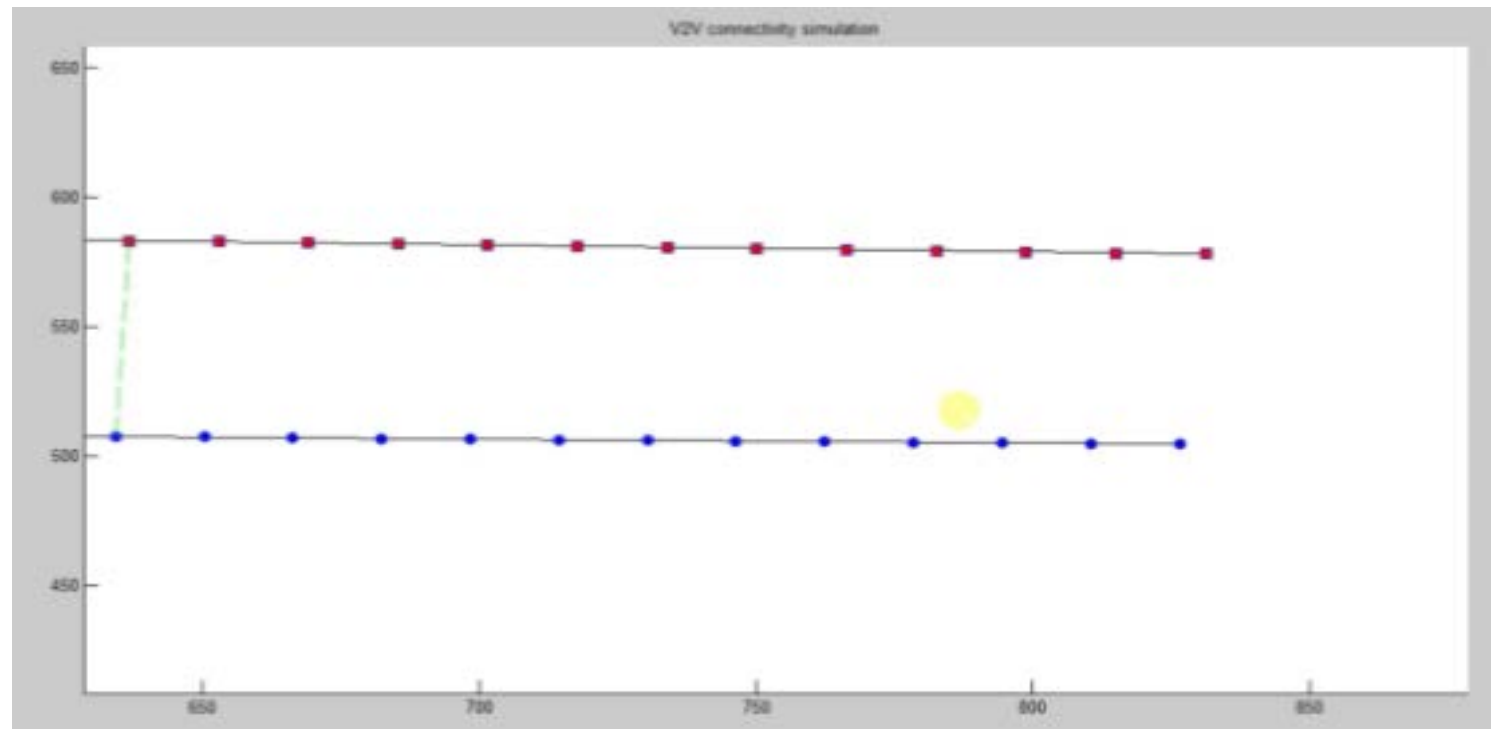

Figure 16. These two roads are parallel to each other 


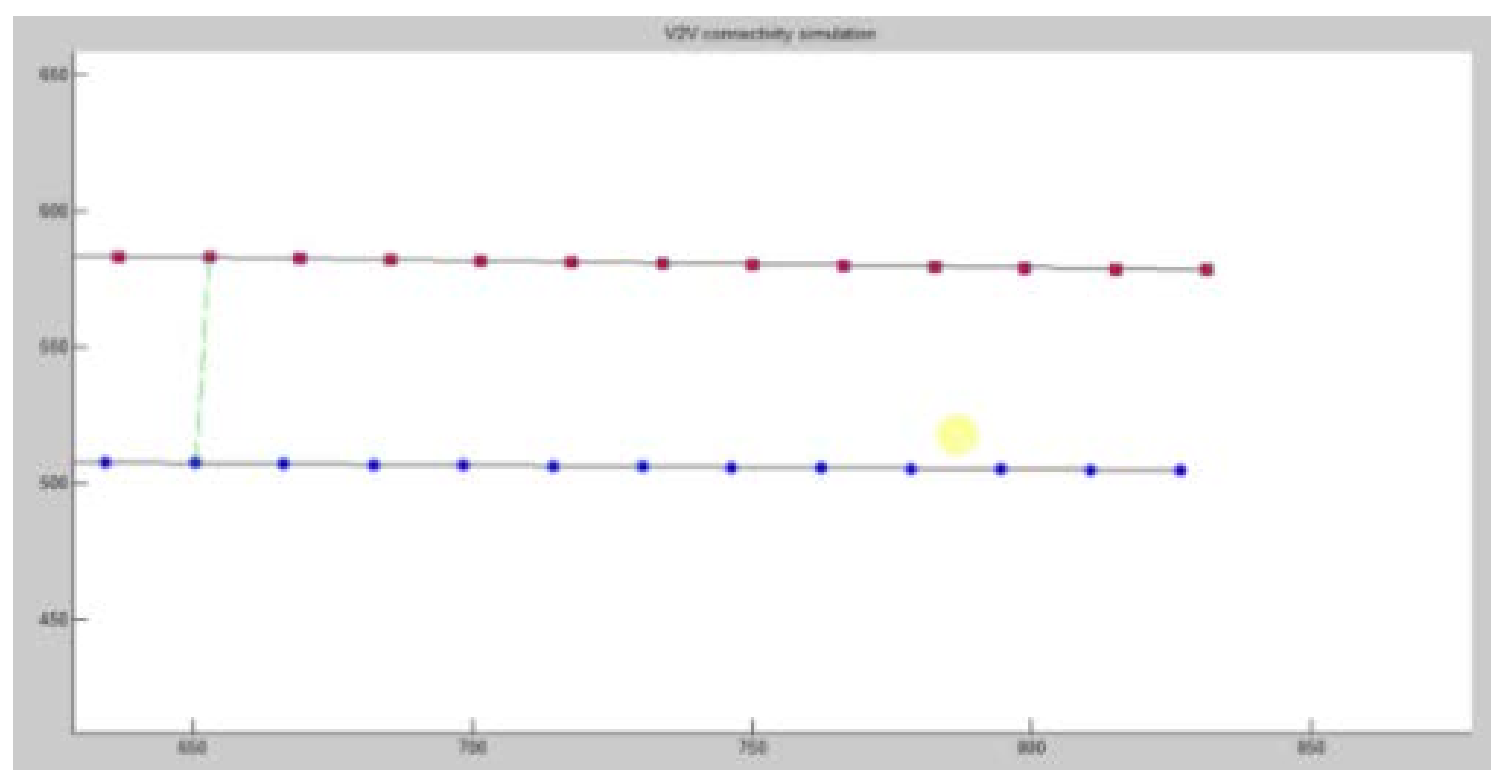

Figure 17. Vehicles are connected with one another but no need to change speed

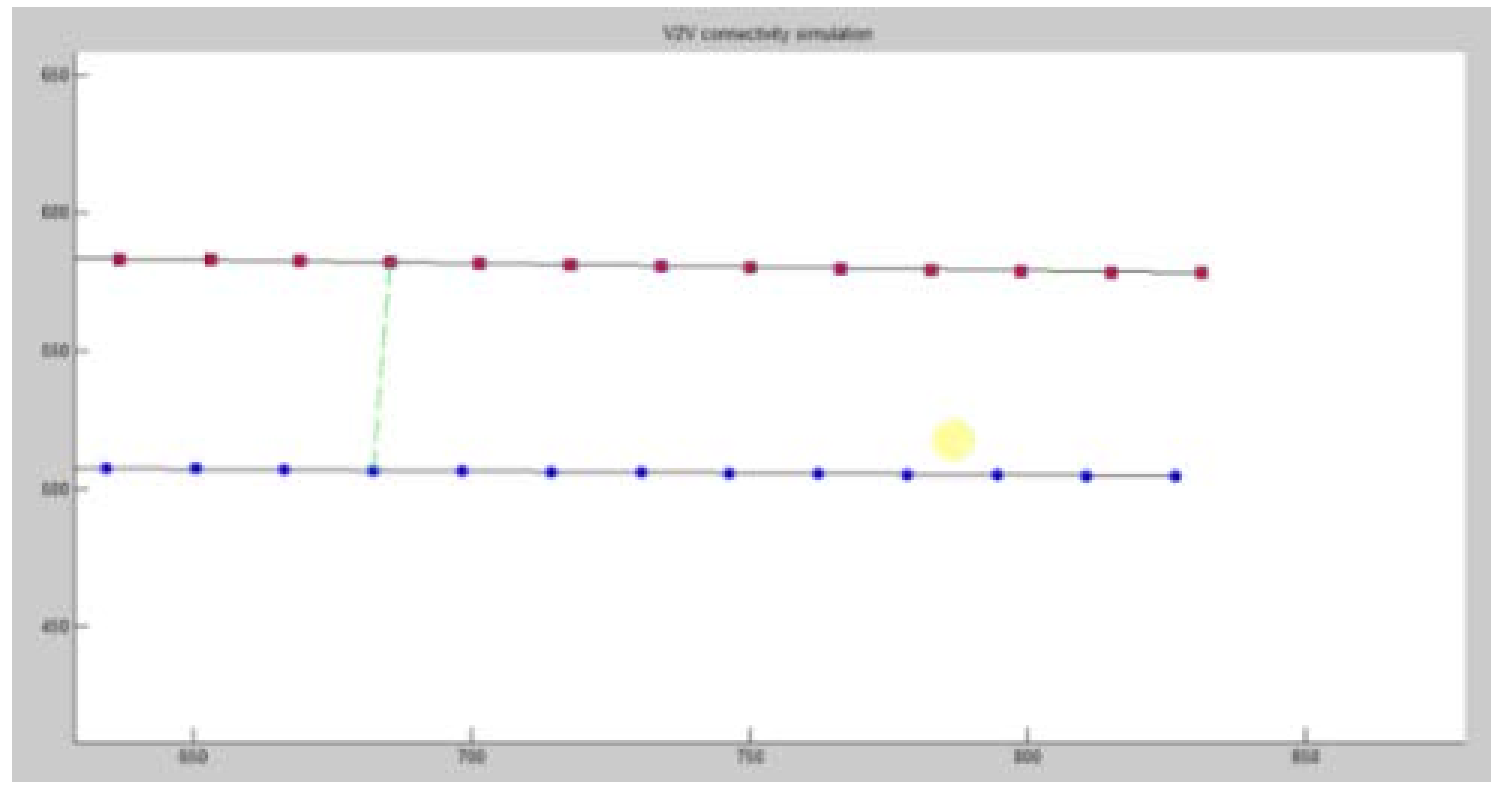

Figure 18. Vehicles are moving continuously

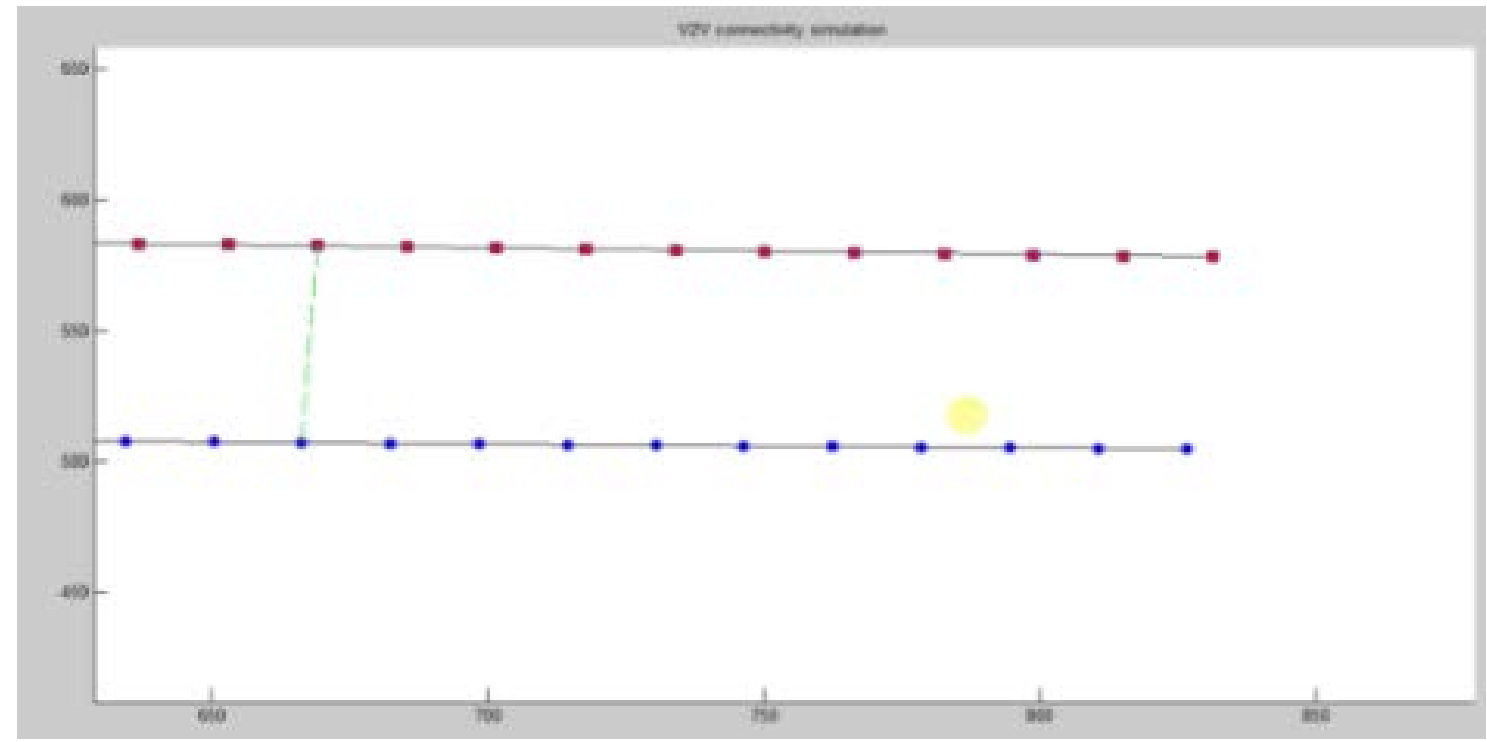

Figure 19. Still connected but no need to change speed 


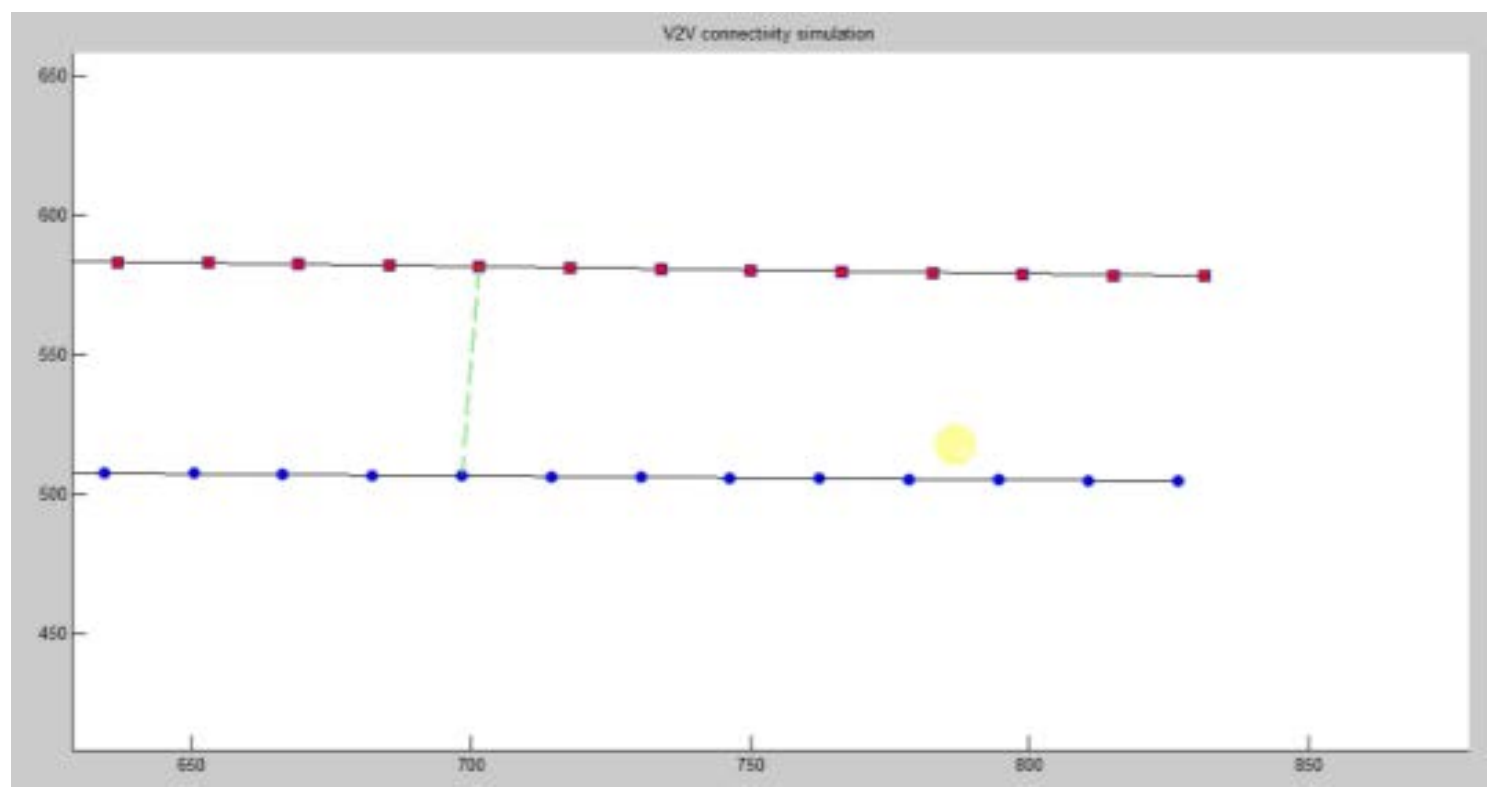

Figure 20. Still connected but no need to change speed

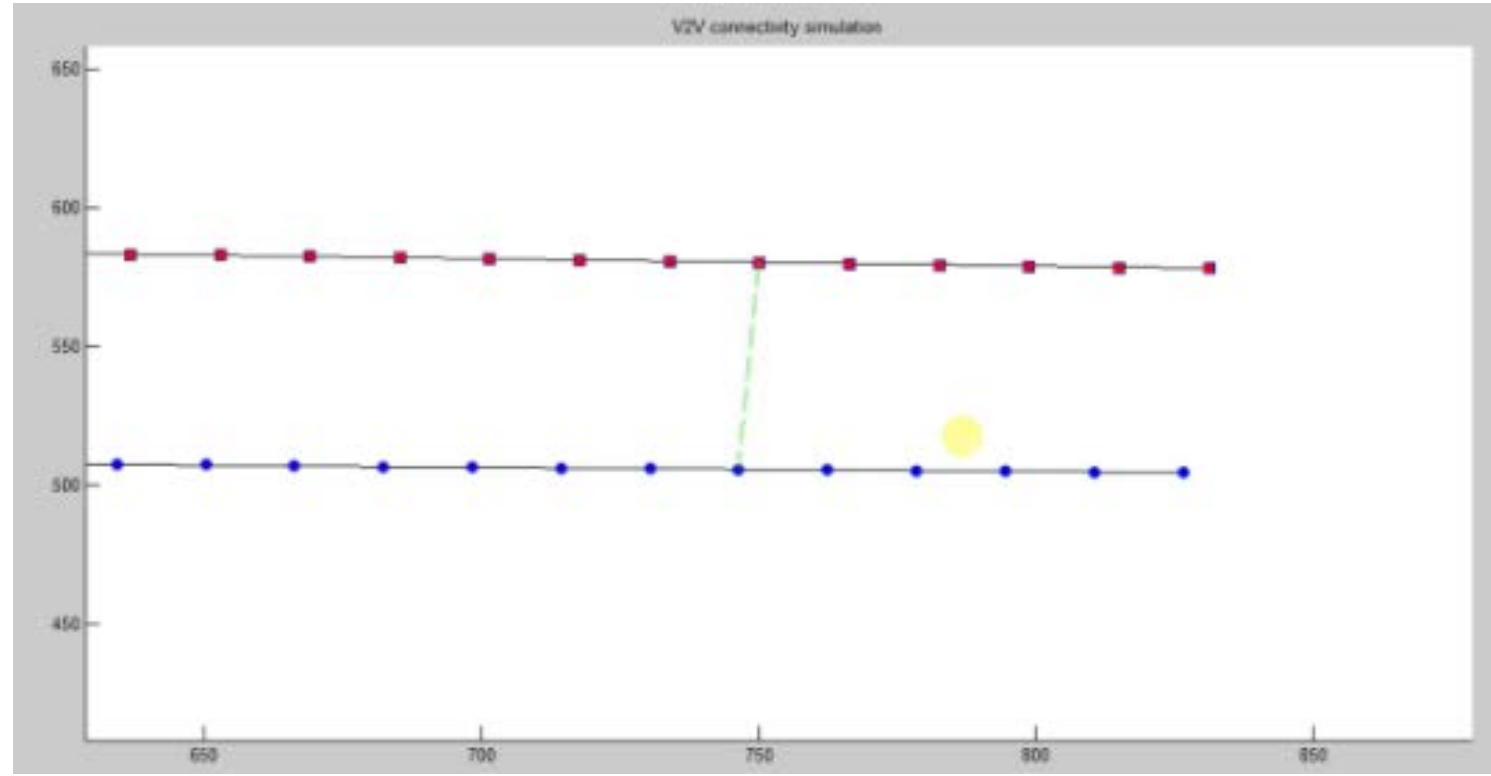

Figure 21. Still connected but no need to change speed

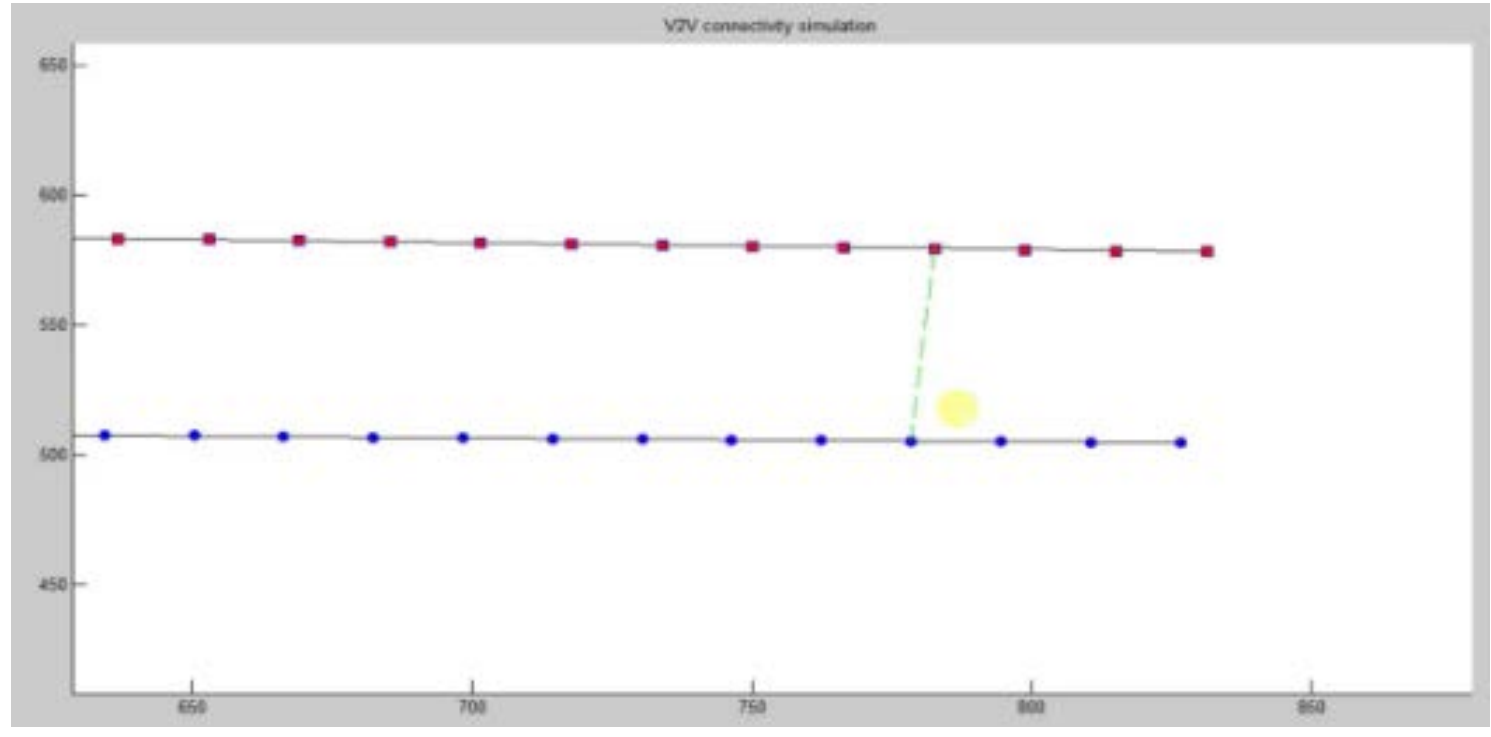

Figure 22. Still connected but no need to change speed 


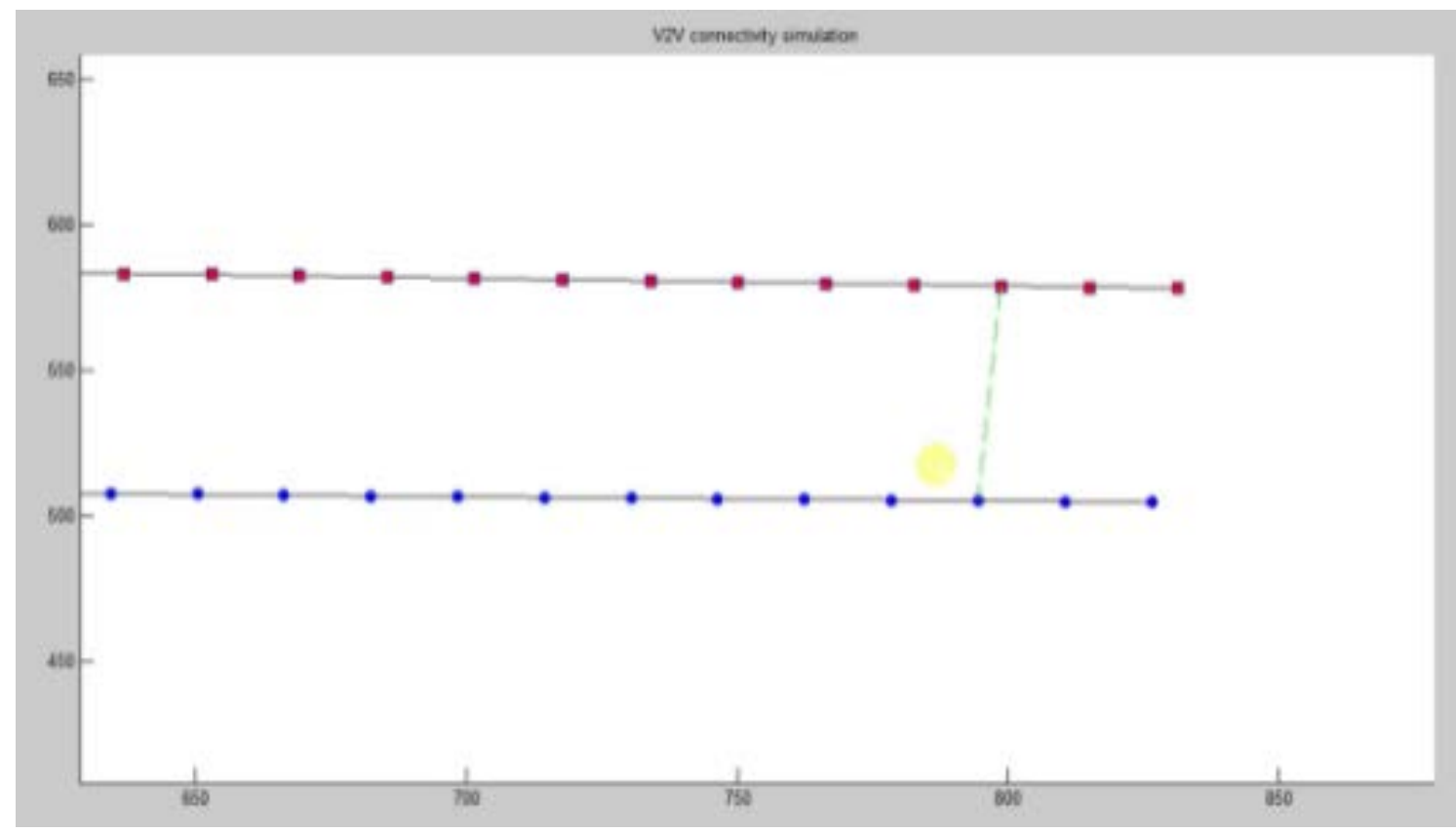

Figure 23. Still connected but no need to change speed

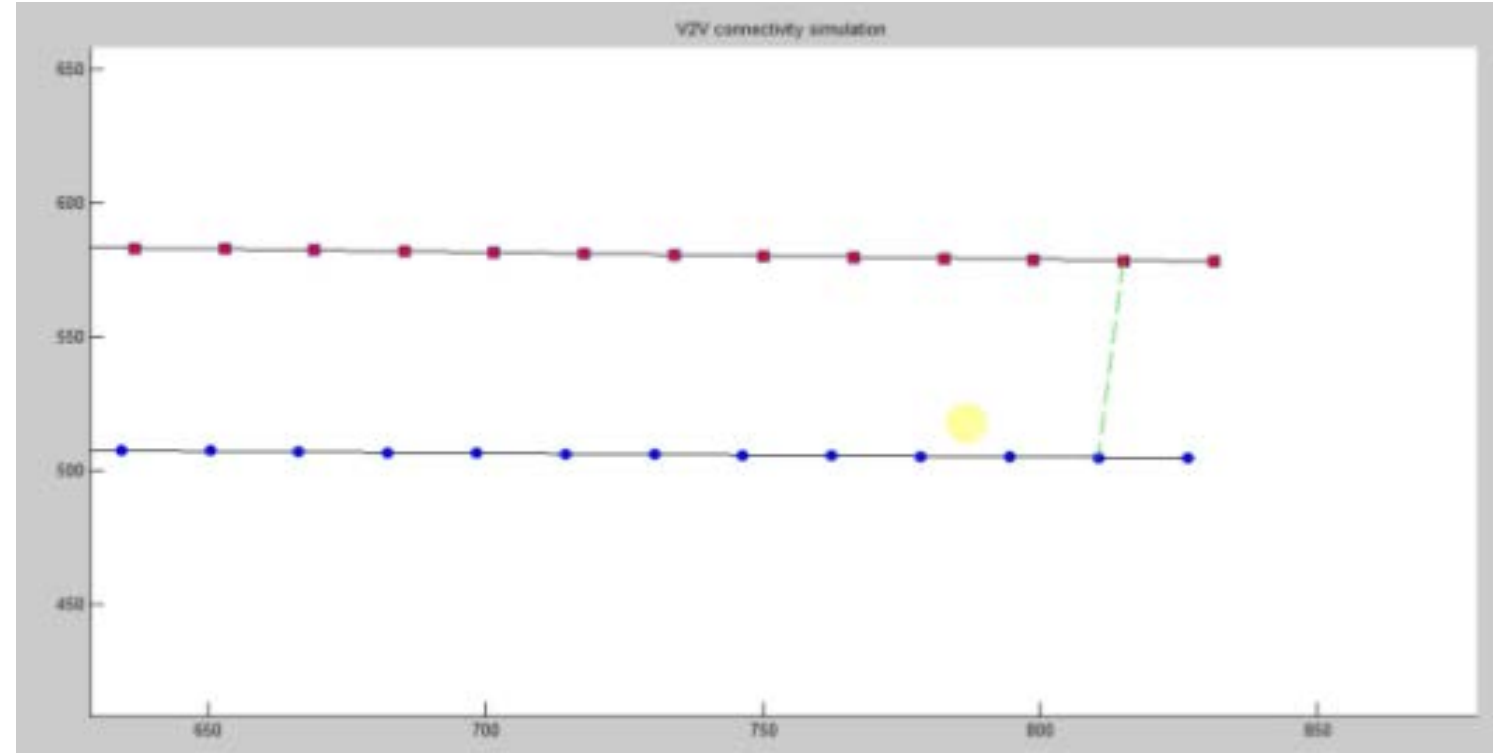

Figure 24. Still connected but no need to change speed

\section{Conclusion}

The simulation shows the importance of communication using the DSRC standard. The simulation also indicates that by using Dedicated Short Range Communication System, traffic flow can improve leading to reduced accidents. However, specific constraints and future research directions are identified for packet routing protocols used to support such applications in the DSRC environment.

As vehicles follow each other's instruction and as one of them becomes slave and other master, it could be seen that vehicles crossed without any collision due to speed difference. Although, DSRC VEHICLESIM was not implemented based on MAT-LAB's decreased functionality on this, the results from the experiment demonstrate the need for network data prioritization for safety-critical applications. The future use of vehicular networks offers a tremendous opportunity for safety measures as DSRC may provide better communication standard for flow of traffic as a result of which congestion of traffic decreases, collisions avoided and crashes stopped.

\section{References}

[1] Armstrong. Lee (2007) Dedicated Short Range Communications (DSRC) home, retrieved from http://www.leearmstrong.com/dsrc/dsrchomeset.htm.

[2] Myung, H. G., Lim, J., \& Goodman, D. J. (2006). Single carrier FDMA for uplink wireless transmission. IEEE Vehicular Technology Magazine, 1(3), 30-38.

[3] Davis, J. P., Eisenhardt, K. M., \& Bingham, C. B. (2007) Developing theory through simulation methods. Academy of Management Review, 32(2), 480-499.

[4] Kenney, J. B. (2011). Dedicated short-range communications (DSRC) standards in the United States. Proceedings of the IEEE, 99(7), 1162-1182. 
[5] Musse, S. R., Jung, C. R., Jacques, J., \& Braun, A. (2007). Using computer vision to simulate the motion of virtual agents. Computer Animation and Virtual Worlds, 18(2), 83-93.

[6] Kosch, T., Kulp, I., Bechler, M., Strassberger, M., Weyl, B., \& Lasowski, R. (2009). Communication architecture for cooperative systems in Europe. IEEE Communications Magazine, 47(5).

[7] Weitfield C. (2003) Simulation techniques for the performance evaluation of DSRC systems; retrieved: http://www.wirelesscommunication.nl/reference/chaptr01/dtmmsy st/dsrc/dssr10.htm.

[8] Nag, A., Biswas, S., Sarkar, D., \& Sarkar, P. P. (2011). A novel technique for image steganography based on DWT and Huffman encoding. International Journal of Computer Science and Security, (IJCSS), 4(6), 497-610.

[9] Richmond Adebiaye (2017). Investigating IT Effectiveness: Perspectives Relative to Cultural Differentiation between IT Users and Service Providers; American Journal of Networks and
Communications (AJNC), Science Publishing Group, 2017; Volume 6, Issue 3, June 2017, Pages: 54-61.

[10] IEEE 802.11e/D4.0 (2002), “Draft Supplement to Part 11: Wireless Medium Access Control (MAC) and physical layer (PHY) specifications: Medium Access Control (MAC) Enhancements for Quality of Service (QoS),” 2002.

[11] Richmond Adebiaye, Haroun Alryalat, Theo Owusu (2016) Perspectives for Cyber-Deterrence: A Quantitative Analysis of Cyber Threats and Attacks on Consumers: International Journal of Innovative Research in Science, Engineering and Technology (IJIRSET) Vol. 5 Issue 7, August 2016. PP 01-18.

[12] J. Zhu and S. Roy (2003). "MAC for Dedicated Short Range Communications in Intelligent Transporation System”. IEEE Communications Magazine, pages 60-67, 2003.

[13] Qing Xu et al (2004) Vehicle-to-Vehicle Safety Messaging in DSRC. Source: DBLP Conference: Proceedings of the First International Workshop on Vehicular Ad Hoc Networks, 2004, Philadelphia, PA, USA, October 1, 2004. 